\title{
Mobilisation identitaire et représentation politique des « Turcs » en Thrace occidentale : les élections législatives grecques de mars 2004
}

Identitarian mobilization and political representativity of "Turks" in Western

Thrace: Greek parliamentary elections in March 2004

Jeanne Hersant et Nepheli Yatropoulos

\section{OpenEdition}

\section{Journals}

Édition électronique

URL : http://journals.openedition.org/ejts/1342

DOI : 10.4000/ejts. 1342

ISSN : $1773-0546$

Éditeur

EJTS

Référence électronique

Jeanne Hersant et Nepheli Yatropoulos, « Mobilisation identitaire et représentation politique des

"Turcs » en Thrace occidentale : les élections législatives grecques de mars 2004 », European Journal of Turkish Studies [En ligne], Liste complète, mis en ligne le 11 janvier 2008, consulté le 19 février 2020. URL : http://journals.openedition.org/ejts/1342 ; DOI : 10.4000/ejts.1342

Ce document a été généré automatiquement le 19 février 2020.

(c) Some rights reserved / Creative Commons license 


\section{Mobilisation identitaire et représentation politique des «Turcs » en Thrace occidentale : les élections législatives grecques de mars 2004}

Identitarian mobilization and political representativity of "Turks" in Western

Thrace: Greek parliamentary elections in March 2004

Jeanne Hersant et Nepheli Yatropoulos

\section{Introduction}

1 La Thrace occidentale fait partie de ces régions peu connues des confins balkaniques de l'Union européenne, dont la vie politique est marquée, directement et indirectement, par la Turquie voisine. Les musulmans, majoritairement turcophones, qui vivent dans la région, ont été exemptés de l'échange obligatoire de populations entre la Grèce et la Turquie par le traité de Lausanne de $1923^{1}$. Ils constituent la seule minorité officiellement reconnue en Grèce, quoique uniquement en tant que minorité religieuse. Or, le traité de Lausanne définissant la Turquie comme puissance garante, et la pratique des autorités grecques dès les années 1930, ont peu à peu conféré à l'État turc de véritables prérogatives, définies en dehors de tout cadre légal, concernant la gestion de cette population (Hersant 2007), exercées par l'entremise du consulat général de Turquie à Komotini (notamment la rémunération d'une partie du personnel religieux et des instituteurs des écoles de la minorité2). La vie politique locale a été marquée, au début des années 1980, par l'émergence d'un mouvement de protestation contre les discriminations à l'encontre des musulmans, devenu par la suite mouvement identitaire " turc $»^{3}$. Les animateurs de ce mouvement ont choisi un répertoire d'action tourné vers les institutions européennes - en usant notamment du registre de la 
protection des minorités "nationales» ou "ethniques" - autant que vers la représentation diplomatique turque de la Thrace. L'un d'entre eux, Sadık Ahmet (1947-1995), premier député indépendant de la minorité, constitue aujourd'hui la figure mythique de ce mouvement et des personnalités qui s'en réclament.

Depuis les années 1990, les travaux universitaires mettant en avant les "problèmes d'identité » de la « minorité musulmane/turque » de Thrace occidentale se multiplient, mais l'approche sociologique est occultée par la perspective interétatique qui est privilégiée, laquelle tend à réifier la question minoritaire comme élément du contentieux gréco-turc ${ }^{4}$. Ainsi, l'action protestataire, les ressorts de la mobilisation identitaire et la participation politique des musulmans ne sont pas pris en compte. Or, depuis la fin des années 1990, dans un contexte de détente politique et d'européanisation, les acteurs de ce mouvement identitaire ont investi le champ politique local, parvenant à imposer leurs propres registres lexicaux et pratiques politiques, lesquels sont tolérés, parfois même repris, par les acteurs politiques "grecs".

3 L'hypothèse examinée ici est celle d'une dualité du champ politique local, auquel s'intègre ponctuellement, en période électorale, ce que nous nommerons une arène publique "turque». Cet article se fonde sur une étude menée dans le cadre des élections législatives de mars 2004, et se propose de montrer que la constitution d'une arène publique "turque", autonome et productrice d'enjeux au sein du champ politique local, a pour contrepartie paradoxale la représentation électorale relativement marginale des musulmans. La Thrace occidentale avait droit cette annéelà à dix députés, dont six pour les deux préfectures [nomoi] du Rhodope et Xanthi ${ }^{5}$, qui ont servi de cadre à cette étude. Or, malgré la proportion de musulmans turcophones, qui représentent plus de la moitié de la population de ces deux départements considérés ensemble ${ }^{6}$, un seul député issu de leurs rangs a été élu dans le Rhodope le 7 mars 20047, et aucun à Xanthi. Ce phénomène se retrouve dans la plupart des élections législatives depuis le retour à la démocratie en $1974^{8}$, à l'exception de celles de 1996, marquées par le caractère récent de la disparition de Sadık Ahmet, qui ont vu l'élection de trois députés « turcs ». L'unique député élu en 2004, İlhan Ahmet, était un novice en politique, qui avait fait un piètre score lors de son entrée sur le marché politique, à l'occasion des élections locales d'octobre 2002 ; fait inédit, il a été élu alors qu'il n'était pas soutenu par le consulat général de Turquie de Komotini. On s'intéressera à l'échec relatif du vote "communautaire" lors de ces élections législatives, en dépit d'une mobilisation massive de l'électorat musulman, illustrant de surcroît l'aspect inédit et l'ampleur de la mobilisation des électeurs issus de cette minorité résidant en Turquie.

Il convient dès à présent de justifier les dénominations dont il sera fait usage ici, qui expriment le scepticisme et la volonté de distanciation des auteures vis-à-vis des catégories utilisées à la fois par les chercheurs et les acteurs. L'emploi de l'adjectif «musulmane » ou «turque » pour qualifier la minorité semble comporter un parti pris implicite, l'État grec ne reconnaissant cette minorité que comme musulmane tandis que l'État turc appuie les revendications du mouvement identitaire revendiquant la reconnaissance de la «turcité » de l'ensemble de la minorité. L'observateur extérieur est d'ailleurs constamment prié de prendre position. Pour notre part, nous avons choisi de parler de musulmans lorsqu'il sera question de façon générale des membres de la minorité, et de « Turcs » lorsqu'il sera question des acteurs politiques ou associatifs qui sont partie prenante au mouvement de revendication identitaire. Constitué en 
ressource sur la scène politique locale, et facteur de reconnaissance sociale pour ceux qui s'en réclament, le discours de la turcité ne saurait pour autant être ramené à une réalité sociale objective. L'usage des guillemets permet en outre de distinguer les "Turcs» de Thrace occidentale des Turcs citoyens de la République de Turquie. Il n'existe pas d'étude quantitative systématique des comportements électoraux en Thrace occidentale sur la longue durée. Elles sont extrêmement localisées (Marantzidis, Mavromatis 2000), ou bien elles se contentent de recenser les scores électoraux obtenus par les principaux candidats " turcs » sur des périodes plus ou moins longues (Akgönül 1999, Aarbakke 2000). Ces données partielles ne permettent pas de mettre les résultats en perspective, et encore moins d'élaborer un système d'explication autour de la participation électorale en Thrace occidentale. La recherche présentée ici ne permettra que partiellement de combler ce manque, il est en effet quasiment impossible d'obtenir des données chiffrées fiables concernant la démographie ou le comportement électoral de la population musulmane de Thrace occidentale.

5 Rappelons que depuis le recensement de 1951 aucune statistique officielle en Grèce ne prend en compte l'appartenance confessionnelle ou la pratique dans la sphère privée d'une autre langue que le grec. Dans la mesure où la population musulmane en Thrace n'est pas connue avec exactitude, nous n'avons donc pu éviter le recours à des estimations concernant l'électorat musulman (voir la présentation de la méthodologie en annexe) $)^{9}$. La collecte de données chiffrées, qui soient suffisamment précises pour pouvoir analyser la répartition des voix par candidat et par bureau de vote, dans les deux départements (Xanthi et Rhodope), n'a pas été facile. Les chiffres fournis par le ministère de l'Intérieur grec étaient parfois incomplets et nous avons dû recourir à certains bureaux politiques locaux ${ }^{10}$. Nous avons certes eu accès à des données non officielles du nombre de votes musulmans enregistrés lors des trois dernières élections législatives, qui nous ont été fournies par des journalistes et personnalités politiques locales; mais là encore, il s'agit le plus souvent d'estimations. Pour pallier cela, nous avons, dans chacun des deux départements, procédé à l'analyse à partir de données exactes sur des échantillons représentatifs: les résultats électoraux de quelques municipalités dont la population est exclusivement musulmane (entre 12000 et 14000 électeurs pour les échantillons concernant Xanthi et entre 14000 et 16000 pour les échantillons concernant le Rhodope). Nous avons choisi de traiter ces données en comparaison avec celles des scrutins législatifs de 1996 et 2000, les élections de 1996 marquent en effet le début d'une nouvelle période puisque aucun candidat "turc " indépendant ne s'est présentée pour représenter la minorité, contrairement à la période 1985-1993.

6 Ces remarques étant faites, il convient de nuancer la portée de nos échantillons. Tout d'abord, ils concernent des régions rurales, parfois isolées, et ne permettent pas de prendre en compte le vote musulman urbain. Or les modes et niveaux de vie sont fortement différenciés entre les villes et leur arrière-pays (Hersant 2007), c'est notamment flagrant au regard de la faible durée de la scolarisation des filles. Notre échantillonnage reflète dès lors des conditions particulières d'exercice du vote, dans un environnement où le contrôle social est très prégnant. Par ailleurs, les chiffres présentés ici ne sauraient à eux seuls rendre compte des déterminants du vote localement ; c'est pourquoi nous avons choisi de ne pas nous concentrer seulement sur l'analyse quantitative du vote, qui ne permet pas d'appréhender en tant que telle les pratiques politiques, ni les dynamiques sociales qui sous-tendent les rivalités et représentations politiques. Rappelons que pour étudier les déterminants du vote, il faut 
envisager le positionnement des agents dans un espace structuré par les clivages politiques et sa traduction en termes de participation électorale (Lagroye et alii 2002). Il n'était matériellement pas possible dans le cadre de cette étude, de mener une enquête qualitative de longue haleine, qui aurait permis d'analyser avec précisions les choix électoraux du groupe considéré ; aussi nous centrerons le propos sur la démonstration suivante: si du point de vue des pratiques politiques, des représentations et de la politisation au sens de " compétence politique » (Gaxie 1978), nos enquêtes mettent en évidence l'intégration des musulmans de Thrace occidentale au système politique grec, il n'est pas possible en revanche d'interpréter simplement les résultats électoraux de 2004, comme l'expression d'une défection de leur part vis-à-vis des candidats « turcs ».

7 La consigne du "vote communautaire » a en effet été largement suivie, comme le montrent les données recueillies; nous nous attacherons à expliquer cela. Notre objectif est de nous démarquer des analyses récurrentes en termes d'appartenance ethnique, de "schizophrénie » identitaire ${ }^{11}$ et d'allégeance à la Turquie. Il s'agit plutôt de saisir le changement opéré en l'espace de quelques années, au début des années 2000 : avec la libéralisation politique en Thrace, le discours de « la minorité opprimée » a changé d'usage ${ }^{12}$ pour devenir localement une ressource politique, et l'affirmation de la turcité une clé d'entrée dans le champ politique local. Pour cette étude seront utilisés des entretiens effectués en Thrace occidentale entre août 2002 et mars 2004, auprès de candidats et de personnes impliquées dans les associations "turques" et la presse locale turcophone. L'une de nous a en outre effectué le voyage en bus depuis Istanbul avec des personnes qui rentraient voter en Thrace. Nous avons complété ces enquêtes par dépouillement du journal turcophone Gündem [Ordre du jour], publié à Komotini, entre août 2002 et septembre 2004. Pour la période des élections, nous avons eu recours à la presse quotidienne de Turquie, ainsi que ponctuellement au journal trilingue Paratiritis, publié à Komotini en grec, en turc et en russe. Dans une première partie, nous décrirons les interactions entre les acteurs étatiques grecs et turcs, ceux du champ politique local et ceux de l'arène publique « turque ». La seconde partie portera spécifiquement sur la campagne électorale, c'est-à-dire les registres d'action et pratiques adoptées, ainsi que les références à la Turquie. Quant à la dernière partie, consacrée à l'analyse des résultats des élections de 2004, elle nous permettra d'isoler les variables explicatives de la sous-représentation électorale des musulmans dans chacun des deux échantillons représentatifs.

\section{Champ politique local et arène publique « turque »}

Considérons la compétition politique en Thrace occidentale - et plus particulièrement dans les départements de Xanthi et du Rhodope - comme constitutive d'un champ politique local au sein duquel s'inscrit ponctuellement une arène publique (Cefaï 2002), ce qui le rend spécifique. Les enjeux qui structurent cette arène, liés à la revendication de la turcité de la minorité, se sont constitués en tant que tels dans la seconde moitié des années 1980, notamment autour de la personnalité du député Sadık Ahmet. Cet espace a acquis son autonomie dans les années 1990, lorsque la libéralisation politique dans un contexte européen a permis la libre expression publique de ses enjeux. Contesté de son vivant pour son discours radical et ses amitiés affichées avec des personnalités politiques de Turquie, il est désormais considéré comme «celui qui a 
ouvert la voie » et il est impossible à quiconque briguant un mandat électif, local ou national, de ne pas se réclamer de l'héritage du héros défunt ${ }^{13}$.

\section{Sadik Ahmet}

Sadık Ahmet est né en 1947 dans le village d'Agra, à $30 \mathrm{~km}$ environ à l'est de Komotini ; il est entré en politique après un échec professionnel : il se vit refuser un poste vacant à l'hôpital de Thessalonique, alors qu'il était le seul candidat. Il se replia alors sur les structures associatives de la minorité et devint le secrétaire de l'Association des diplômés de Thrace occidentale, à Komotini. À la même époque, les dirigeants de l'association des Turcs de Thrace occidentale de Düsseldorf eurent l'idée de lancer une campagne de signatures pour dénoncer le traitement infligé aux membres de la minorité, particulièrement après la crise de Chypre. Au cours de l'année 1986, Sadık Ahmet prit l'initiative de collecter des signatures à la faveur de ses tournées dans les villages, où il pratiquait les circoncisions: il en obtint un millier environ. Le 9 août 1986, il fut interpellé pour excès de vitesse sur l'autoroute d'Alexandroupolis (Dedeağaç) ; les policiers découvrirent de nombreux exemplaires de la pétition dans le coffre de sa voiture et l'arrêtèrent. Il fit ainsi un premier séjour en prison. En janvier 1990, Sadık Ahmet et İbrahim Şerif (l'actuel mufti dissident de Komotini) furent arrêtés et jugés pour avoir utilisé le mot « Turc » dans leurs tracts et articles publiés au cours de la campagne électorale de novembre 1989. Ce verdict a donné lieu à des manifestations et affrontements; la tension fut telle que le consul turc de Komotini et le consul grec d'Istanbul furent chacun reconduits à la frontière les jours suivants. Sadık Ahmet créa son propre parti en 1991, le Parti de l'amitié, de l'égalité et de la paix [Dostluk, Eşitlik ve Barış Partisi $\mathrm{DEB}]$, qui n'a qu'une existence symbolique en raison du barrage électoral introduit en 1990. Lors des différents scrutins auxquels il se présenta, Sadık Ahmet obtint un score jamais égalé par aucun candidat musulman. Il fut également celui qui a officialisé les liens avec le «monde turc ", c'est-à-dire les représentants de la droite nationaliste et radicale turque, dont le discours est en partie fondé sur ceux qu'on appelle les "Turcs de l'extérieur» [dış Türkler], dont le cercle le plus proche englobe les Turcs de Thrace occidentale et ceux de Chypre. Sadık Ahmet est décédé dans un accident de voiture le 25 juillet 1995, devenant ainsi le martyr des "Turcs » de Thrace occidentale. Il est la première personnalité qui ait à la fois saisi la Cour européenne des droits de l'homme, en 1993, tout en affichant ses liens avec la droite ultranationaliste de Turquie.

\section{Définir l'arène publique « turque » en Thrace occidentale}

9 La présente étude s'inscrit dans une réflexion plus vaste sur les ressources politiques et sociales liées à l'affirmation de l'identité «turque » de la «minorité musulmane » de Thrace occidentale, depuis la fin des années 1980, au sein du champ politique local dominé par les partis politiques "grecs", mais aussi à l'échelle européenne. La mobilisation des «Turcs » de Thrace occidentale dans un cadre européen a co-émergé dans l'espace migratoire; on peut en retracer les enchaînements, depuis les années 1980, de la Turquie à l'Allemagne et à la Thrace occidentale (Hersant 2007). S'est alors constituée ce qu'on peut appeler une arène publique des "Turcs» de Thrace occidentale, au sein de laquelle prend place le registre européen des droits de l'homme, registre sémantique et registre de légitimation coexistant avec celui du nationalisme turc. La notion d'arène publique, telle que définie par Daniel Cefaï $(2002: 53,69)$, paraît en effet le mieux à même de qualifier l'espace des pratiques, revendications, ainsi que les référents idéologiques et symboliques des « Turcs » de Thrace occidentale, en Grèce et dans l'espace migratoire (notamment en Allemagne et Turquie). Cette arène transnationale s'inscrit dans différents champs: le champ politique local en Thrace occidentale, le champ du pouvoir d'État ${ }^{14}$ en Turquie ou le champ des organisations 
internationales, au sein duquel elle tente d'inscrire ses propres organisations et enjeux (Hersant 2007).

Cet espace de la " turcité » présente a priori les caractéristiques d'un champ : une lutte de position à l'intérieur d'un espace concurrentiel dont les enjeux lui sont propres, et constituent eux-mêmes l'objet d'une lutte d'influence et d'autorité. L'accès à cet espace symbolique a un coût: posséder un certain capital social ou culturel (acquis par diplôme), maîtriser les lignes de démarcation symboliques qui séparent les "Turcs » des «Grecs ", ainsi que les grands moments de l'histoire officielle ; c'est-à-dire prêter allégeance à la mémoire de Sadık Ahmet et aux principes du nationalisme turc, tout en s'exprimant selon le registre lexical caractérisé comme étant celui de l'Union européenne et des droits de l'homme. Cette arène bénéficie aussi, de fait, d'une certaine autonomie et fermeture, par l'usage de la langue turque aujourd'hui largement autorisé en Grèce et non accessible à la plupart des acteurs politiques, mais aussi par des pratiques d'exclusion symbolique de ces derniers ${ }^{15}$. Pourtant, ce n'est pas un champ politique au sens conventionnel ${ }^{16}$, dans la mesure où les acteurs qui y évoluent ne participent pas tous à la compétition politique; d'ailleurs aucune organisation politique $^{17}$ ne structure cet espace, les candidats "turcs » aux élections ne disposent pas de leurs propres organisations politiques; ils briguent un mandat électif au sein des formations politiques "grecques». D'autre part, cet espace rassemble les enjeux conventionnellement associés au champ politique et ceux qui relèvent du religieux : les muftis dissidents sont considérés en Thrace comme des représentants politiques ${ }^{18}$. En outre, ce n'est pas un espace réellement concurrentiel : s'il y a bien des enjeux de position au sein de chacune des scènes constituant cet espace (Thrace occidentale, Allemagne, Turquie), il n'y a pas à proprement parler de lutte pour la définition des enjeux. Ceux-ci sont énoncés et arbitrés depuis la Turquie, par les acteurs associatifs, politiques ou étatiques; toute mise en cause de cette définition officielle se traduit par l'exclusion de cet espace de référence. Les acteurs étatiques turcs présents en Thrace occidentale participent à la définition des enjeux qui constituent la scène locale de l'arène, et à la sélection des leaders habilités à en faire partie.

\section{Les principaux acteurs}

Les insiders de l'arène sont tout d'abord les membres du Conseil consultatif de la minorité « turque » [Batı Trakya Türk Azınlı̆ı Danışma Kurulu], qui a vu le jour en 1988 après l'interdiction des associations portant l'adjectif « turc » dans leur nom ${ }^{19}$, dans un contexte politique troublé. Il comprend les muftis dissidents, les présidents de ces associations, ainsi que les élus locaux et députés de la minorité, ces derniers étant positionnés simultanément dans l'arène « turque » et au sein du champ politique local. Le Conseil consultatif est en somme une structure informelle - elle n'a jamais eu la moindre existence juridique - qui rassemble les notables et les personnes autorisées à émettre une opinion sur les "problèmes de la minorité ${ }^{20}$, lesquels se partagent les postes à responsabilité au sein des structures associatives, mais aussi les mandats électoraux ne serait-ce que localement ${ }^{21}$. La cooptation des acteurs politiques " turcs " se fait au sein de ce Conseil consultatif, en bonne entente avec le consul général de Turquie ; ce sont eux qui représentent la minorité à l'extérieur. La proximité culturelle avec la Turquie est par ailleurs cultivée à travers les activités associatives, notamment celles qui ont trait à la valorisation d'un folklore local. 
12 À côté des associations interdites représentées au sein de ce Conseil, figure l'Association des diplômés d'université de la minorité en Thrace occidentale, qui est la seule à fonctionner officiellement, car elle est la seule dont l'intitulé ne comporte pas l'adjectif 'turc'. Sa création en 1982 visait à aider à l'insertion professionnelle des jeunes musulmans ayant fait leurs études en Turquie, mais aussi à fédérer les musulmans bénéficiant d'un capital universitaire contre la mainmise du consulat turc sur la vie sociale et politique locale. Sa position a évolué par la suite, notamment avec l'interdiction, au cours des années 1980, des autres associations ; elle joue désormais un rôle clé dans la représentation des «Turcs » de Thrace aussi bien dans les congrès du «monde turc» que dans les différents forums européens de promotion des droits des minorités. De ce fait, les postes de direction au sein de cette association sont devenus des objets de convoitise, voire de tractations avec le consulat turc en période électorale. Le Conseil consultatif de la minorité accueille également parmi ses membres Ahmet Haciosman, le président du Parti de l'amitié, de l'égalité et de la paix [Dostluk, Eşitlik ve Barış Partisi, DEB] $]^{22}$, le parti politique fondé par Sadik Ahmet en 1991 pour essayer d'asseoir son leadership et se démarquer des autres notables. En raison du barrage de 3 $\%$ des voix lors des scrutins législatifs, ce parti a seulement participé au scrutin de 1993, en n'ayant aucune chance de voir ses candidats élus; ce n'est plus aujourd'hui qu'une formation symbolique. Les personnes impliquées dans les associations, membres ou non du Conseil consultatif, mais aussi les journalistes de la presse turcophone sont en somme les principaux acteurs de l'arène publique « turque » en Thrace occidentale.

\section{Les outsiders}

13 Considérant que le discours de la turcité est la clé d'entrée au sein de l'arène publique " turque ", certains acteurs en sont à la lisière et y ont un droit d'entrée en reprenant ponctuellement ce discours. Il s'agit notamment de Mehmet Ahmet et Mustafa Mustafa qui, eux, ont un parcours militant de longue date au sein d'un parti politique, le PASOK pour le premier, le KKE puis le Synaspismos pour le second ${ }^{23}$. Il existe en effet un système parallèle d'allégeance politique au sein de la "minorité ", qui dénote une intégration réussie de nombre d'acteurs politiques musulmans au système politique grec, alors que pour les acteurs politiques « turcs » l'appartenance à un parti politique " grec » n'est qu'un moyen de briguer un mandat électif. Mécanicien de profession, Mehmet Ahmet est membre du PASOK depuis sa création en 1974; depuis 1977 il a toujours été investi candidat de ce parti. Il a été élu député du Rhodope en 1981, et en 2000 il a obtenu un siège au Parlement suite à une procédure engagée contre sa colistière, Hrissa Manolia, qui n'avait pas démissionné des autres charges publiques qu'elle détenait après avoir été élue. Mustafa Mustafa est médecin, il a été candidat à toutes les élections législatives depuis 1981 au sein du Synaspismos, mais n'a été élu qu'une seule fois, en 1996. Il est en revanche la seule personnalité « turque » qui puisse se prévaloir d'un électorat mixte en tant que secrétaire régional de son parti ; en 1996 et en 2000, le Synaspismos conduit par Mustafa Mustafa avait obtenu dans le Rhodope un score nettement supérieur à celui totalisé au niveau national ; le Rhodope constitue à ce titre un département clé pour l'entrée du Synaspismos au Parlement même si pour la première fois en 2004, il y a réalisé un score inférieur à sa moyenne nationale. Ahmet Mehmet et Mustafa Mustafa se conforment au moins partiellement au discours dominant - particulièrement en période électorale - pour ne pas être exclus de l'arène «turque ». Ils sont membres du Conseil consultatif de la minorité en tant qu'anciens 
députés; Ahmet Mehmet l'a présidé entre 2003 et 2004. Mustafa Mustafa est en outre membre de l'Association des diplômés, dont il a été secrétaire général dans les années 1980.

Les outsiders sont ceux qui participent à la compétition politique sans reprendre le discours et le lexique dominants au sein de l'arène «turque ». Le terme d'outsider doit donc ici être compris dans son sens immédiat; néanmoins on ne saurait l'utiliser sans avoir à l'esprit la définition de Howard Becker relative à la déviance (Becker 1985 : 25). Le phénomène observé ici relève a priori plutôt de la transgression d'un principe rhétorique (le discours de la " turcité ») que de celle d'une norme, mais la systématicité de ce principe et son rôle dans la sélection de l'élite de la minorité l'élèvent au rang de normes. En 2004, les bulletins collectés à la sortie des bureaux de vote indiquaient que le nombre de candidats musulmans était bien plus important que les dix candidats «turcs » présentés dans la presse turcophone locale, dont la liste a été reprise dans la presse de Turquie. Il y avait en réalité une dizaine de candidats musulmans dans chaque département (Xanthi et Rhodope), et non pas pour toute la Thrace. La plupart de ces candidats figuraient sur la liste de partis tellement marginaux ${ }^{24}$ que leur engagement ne peut résulter que d'une adhésion aux valeurs de ces partis. A contrario, le fait que ces candidatures soit passée sous silence par les acteurs « turcs » localement dominants nous renseigne sur le mode de structuration et le degré de fermeture de l'arène "turque »: ces candidats représentent un ferment de division et un risque supplémentaire de dispersion des votes musulmans.

\section{Affiliations partisanes et processus d'investiture}

15 Lors des élections législatives, les partis politiques grecs choisissent de porter la candidature d'une personnalité ayant émergé préalablement au sein de cette arène, tandis qu'en amont les acteurs étatiques valident les candidatures « turques ».

\section{L'intervention des autorités dans la désignation des candidats « turcs »}

Quoique citoyens grecs, les musulmans de Thrace demeurent sous la tutelle du ministère des Affaires étrangères. Le Bureau des affaires culturelles du Rhodope est l'un des organes de liaison du MAE grec ${ }^{25}$; il est plus connu sous le nom de «bureau 105 », qui désigne le numéro du bureau de la préfecture à Komotini où se réunissent, avant les élections, le représentant du Bureau des affaires politiques de la minorité (qui se trouve, lui, à Xanthi), un représentant du ministère de l'Intérieur, un représentant de l'état-major militaire, le gouverneur général de la Thrace occidentale, un représentant des services secrets (KYP), et aussi un représentant de l'Inspection des écoles de la minorité. Les autorités grecques se réservent le droit de déclarer irrecevables certaines candidatures "turques ", après concertation entre les acteurs institutionnels et les acteurs politiques, et en consultant éventuellement le consul général de Turquie. L'argument de la raison d'État prévaut sur les rivalités politiques entre partis, et il s'inscrit dans une continuité qui dépasse les gouvernements successifs. Dans un second temps, sur la base de cette "présélection", les dirigeants des deux grands partis, le PASOK et la ND, entament les négociations préalables aux investitures sur la base de la règle, aujourd'hui institutionnalisée, selon laquelle les listes de quatre candidats doivent comporter deux candidats «turcs » dans le Rhodope et un à Xanthi. Les petits 
partis, qui présentent des listes de une à trois personnes, échappent à cette règle et ne la suivent qu'en fonction des disponibilités des candidats.

Selon İlhan Ahmet, seul député « turc » élu en 2004 « ce bureau [des affaires politiques] dispose de fonds du ministère des Affaires étrangères, qui sont distribués à différentes personnes selon les nécessités. Comme le consulat [turc] distribue des fonds de la même façon. Et pendant les élections ces fonds sont importants $»^{26}$. Le rôle de ces structures étatiques nous permet de nuancer l'ouverture du champ politique local. Deux logiques s'affrontent en effet depuis les années 1990: une logique politique et électorale d'ouverture, et une logique sécuritaire appuyée sur l'idée de raison d'État, pour laquelle les musulmans constituent une menace potentielle, particulièrement en raison de la tutelle dont bénéficie le consulat général de Turquie sur une partie de cette population. Cette tutelle résulte pourtant non pas du traité de Lausanne, mais d'une dévolution de compétences dans la gestion de certains aspects de la vie sociale de ce groupe minoritaire au profit des autorités turques (Hersant 2007). Parallèlement à cela, le Consulat général de Turquie donne son accord ou non aux candidatures "turques", mais n'intervient plus pour en censurer certaines comme par le passé ; d'autant que les rapports gréco-turcs, tout comme ceux de la minorité avec son environnement « grec », ainsi que les enjeux électoraux sont pacifiés. Le travail de parrainage ou de refus des candidats se fait a posteriori par le Conseil consultatif, notamment dans les colonnes de Gündem ${ }^{27}$.

\section{Négociations et allégeances partisanes}

18 Pour ce qui est des affiliations partisanes, mentionnons en premier lieu un phénomène propre à la vie politique grecque, le transfert d'un parti à l'autre. Rare en France, ce type de transaction est répandu en Grèce (comme en Turquie). Ainsi, à l'approche des élections du 7 mars, plusieurs personnalités de premier plan ont fait défection du PASOK pour rejoindre la ND et vice-versa, ou encore du Synaspismos vers le PASOK ${ }^{28}$. À l'échelle de la Thrace et des acteurs "turcs ", le phénomène est d'autant plus présent que le fait de compter des candidats "turcs» est un atout pour les partis grecs. En retour ceux qui veulent se lancer dans la course aux suffrages savent qu'ils pourront sans trop se discréditer aller frapper à la porte de plusieurs partis, puisque telle est la règle du jeu. Ainsi, les deux grands partis grecs PASOK et Nouvelle Démocratie drainent chacun leur lot de fidèles, mais il s'agit souvent moins d'affiliation partisane que de loyautés interindividuelles liées à des intérêts communs.

19 L'ouverture récente du jeu politique se traduit par la concurrence de plusieurs candidats « turcs " pour une même investiture, particulièrement à Xanthi où les partis ne sont tenus qu'à un seul candidat "turc ", laquelle se négocie directement avec les partis politiques, dès lors que les autorités grecques ne l'ont pas censurée, et à condition d'être en mesure de payer pour cela : pour être candidat à l'investiture du PASOK ou de la ND, il fallait en 2004 verser 1000 euros au parti sollicité2 ${ }^{9}$. Les candidats doivent faire jouer leurs propres réseaux locaux de connaissance : İlhan Ahmet, par exemple, a entamé des négociations avec la Nouvelle Démocratie après avoir été introduit et proposé au parti par Euripis Stilianidis, député ND du Rhodope depuis 2000 (nommé vice-ministre des Affaires étrangères à l'issue du scrutin de mars 2004). On peut également citer le cas d'un autre de nos enquêtés, İbrahim Onsunoğlu ${ }^{30}$, qui en 1990 obtint l'investiture de la Nouvelle Démocratie grâce à sa stature locale et à son réseau d'interconnaissances, alors qu'il était un ancien militant d'extrême gauche puis 
membre du PASOK ; la personnalité qui soutint sa candidature dans le Rhodope avait des relations de parenté avec «celui qui décidait au niveau national $»^{31}$. Le rapport des candidats «turcs » aux partis politiques est complexe, d'une part du fait du clivage symbolique entre «Grecs» et «Turcs» constamment réaffirmé dans leur discours; d'autre part en raison de la législation grecque, qui induit un rapport « instrumental » aux organisations politiques: les candidats d'un parti ne sont pas tenus d'être effectivement membres de ce parti ${ }^{32}$. Ainsi Nazmi Ahmet, candidat du KKE dans le Rhodope en 2002 et en 2004, affirmait à un journaliste de Gündem l'interrogeant sur son affiliation partisane : «Je ne suis pas membre du KKE, d'ailleurs je ne suis pas communiste »" Quant à la principale figure politique locale, jusqu'en 2004, le député du PASOK Galip Galip (1996-2004), il est l'héritier d'une dynastie locale ayant obtenu l'investiture de son parti grâce à son nom et ne respectant la discipline de parti qu'à l'approche des élections.

Pour autant, dans une situation de rareté de certains biens ou ressources (emploi pour les musulmans formés en Turquie, permis de conduire et de construire jusqu'aux années 1990 en raison d'une discrimination institutionnalisée), les partis politiques grecs sont des atouts précieux pour leurs candidats comme pour leurs militants. Dans les années 1990 particulièrement, les partis grecs essayaient de concurrencer les candidats "turcs " indépendants par des avantages en nature qu'ils étaient seuls à pouvoir distribuer, grâce à leurs relais dans les différentes administrations : une fois investi par la Nouvelle Démocratie, İbrahim Onsunoğlu s'était vu confier « un certain nombre de permis de conduire pour les véhicules personnels, de fonction, les tracteurs, etc. pour les donner à des électeurs potentiels $»^{34}$. Par ailleurs, quoique minoritaires, nombre de musulmans ont des attaches autres que circonstancielles avec des partis politiques grecs. Le militantisme et l'ascension au sein des partis ont toujours constitué un facteur d'intégration sociale, sinon politique, pour les musulmans comme pour les autres citoyens grecs, dans un système fondé en grande partie sur le clientélisme ${ }^{35}$. Candidats ou non à un mandat électif, ceux qui sont membres de longue date d'un parti politique bénéficient d'avantages, d'autant que la rareté de certaines autorisations administratives, on l'a vu, mais aussi des postes dans les écoles de la minorité conduisent les partis à s'en saisir pour récompenser ceux qui leur sont fidèles.

\section{La campagne électorale et les ressorts de la mobilisation communautaire}

21 Il a été montré ailleurs, à l'appui d'une étude sur les élections locales en Thrace occidentale, comment l'arène publique "turque » contribue à la définition des enjeux de la compétition politique locale, entraînant une recomposition locale des clivages inter et intra partisans (Hersant 2007). Pour les élections municipales et préfectorales, en effet, la coopération entre "Grecs » et "Turcs » est nécessaire, d'autant que les dispositions liées au scrutin local ne permettent pas aux musulmans d'être élus aux échelons les plus élevés, c'est-à-dire aux fonctions de maire (pour les municipalités) et préfet de département. Pour les élections législatives, en revanche, il faut avant tout, pour les acteurs du mouvement identitaire, faire élire des députés "turcs » au Parlement, et la compétition joue alors entre « Grecs » - accusés de détourner les votes musulmans à leur profit - et "Turcs », indépendamment de toute affiliation partisane. De même, à la différence de ce qui se passe lors des élections locales pour les candidats 
des partis de gauche, les candidats "grecs » ne se sentent pas tenus de reprendre les thèmes de campagne liés à la turcité ; ce sont les candidats "turcs" qui doivent adopter un double discours, l'un destiné à leur électorat musulman, l'autre destiné au parti qu'ils représentent. Cela n'empêcha pas les candidats grecs de faire campagne dans les associations « turques » (officiellement interdites) ou de diffuser leurs tracts et programmes en turc. Au niveau national également, la Thrace occidentale est de plus en plus présente dans le débat public. Pour la première fois lors des élections législatives d'avril 2000, la région avait fait l'objet de déclarations et promesses des principaux candidats en lice, en raison de son retard de développement; pour la première fois également, la Turquie n'avait pas cristallisé les débats en tant que menace (Hersant 2000). En 2004, étant donné le faible écart entre les deux grands partis grecs dans les intentions de vote, chacun d'entre eux s'est efforcé de faire basculer l'électorat musulman de Thrace occidentale en sa faveur en intégrant les enjeux « turcs » et la Turquie dans sa campagne.

\section{Les thèmes de campagne dominants au sein de l'arène " turque »: minimiser l'intégration au système politique grec}

Il est en effet très vite apparu que le résultat dans la région serait orienté par les nombreux électeurs venus de Turquie, et que cela pourrait faire basculer la victoire vers l'un ou l'autre camp ${ }^{36}$. C'est également ce que releva la presse de Turquie, qui ne fut pas en reste pour inciter les musulmans de Thrace occidentale résidant en Turquie à " accomplir leur devoir » en allant voter en Grèce ${ }^{37}$. Ainsi, alors que Yorgos Papandreou a fait un geste en démarrant sa campagne électorale par un mea culpa en Thrace occidentale, Konstantin Karamanlis, quelques semaines plus tard, s'est affiché aux côtés du Premier ministre turc Recep Tayyip Erdoğan lors d'un meeting de l'AKP ${ }^{38}$ en vue des élections municipales turques du 28 mars 2004. Pour la première fois également, les acteurs étatiques turcs étaient divisés sur le parti à soutenir : le ministère des Affaires étrangères turc avait développé durant les années précédant les élections des liens étroits avec l'équipe du ministre Yorgos Papandreou, élu nouveau dirigeant du PASOK quelques semaines avant les élections. De ce fait Galip Galip, le candidat du PASOK dans le Rhodope, avait le soutien du consulat général de Turquie à Komotini, du ministère turc des Affaires étrangères, et du Conseil consultatif de la minorité. En tant que candidat de la Nouvelle Démocratie, İlhan Ahmet a obtenu quant à lui auprès du gouvernement turc et de l'AKP le soutien qui lui manquait localement de la part des autorités turques. Ce phénomène est aussi un avatar de la pacification des enjeux liés à la Thrace occidentale dans le cadre des relations gréco-turques, qui se banalisent en tant qu'enjeux électoraux. Cela ne doit pas pour autant occulter le fait que, dans chacun des pays concernés, la Thrace occidentale et sa minorité continuent de faire partie des "questions nationales", au sujet desquelles la raison d'État en Grèce ou l'intérêt national en Turquie sont constamment invoqués.

\section{L'injonction au vote communautaire et la recherche d'un leader charismatique}

Cette configuration liée à des enjeux partisans n'a pas manque dé complexifier une campagne qui se voulait justement, pour les candidats "turc», l'affirmation du fait minoritaire et le rejet du fait partisan, accuser de "diviser » la minorité. En période d'élection, le discours de la turcité revêt la forme d'une injonction du Conseil 
consultatif de la minorité pour que les électeurs musulmans votent «pour leurs propres candidats "; les consignes de vote visent à entretenir le clivage " eux/nous ", présentée comme indépassable, entretenant le registre lexical de l'unité et de la probité. Rıza Kırlıdökme, éditorialiste à Gündem ${ }^{39}$, affectionne particulièrement l'expression de "famille minoritaire» [azınlı ailesi], à qui il prête des vertus de " pureté » [temiz Türk halkl[; le vote est "sacré » [kutsal], il faut l'utiliser à bon escient. Un autre thème privilégié : la compétition électorale ne doit pas désunir la « Minorité » [Azınlik], souvent commodément désignée uniquement comme telle en jouant sur l'opposition tout aussi vague avec la «majorité » [çoğunluk], opposition pratique puisqu'elle évite le recours à des qualificatifs ethniques; elle est pour cela fréquemment utilisée également par les acteurs politiques «grecs». Le registre lexical utilisé par les candidats et commentateurs «turcs» fait abondamment référence aux « manœuvres » ou « combines grecques» [tezgah kurmak], auxquelles s'oppose "l'union et la concorde" [birlik ve beraberlik] des candidats et électeurs "turcs ", selon la formule consacrée du nationalisme turc ${ }^{40}$. Il est d'ailleurs de règle que les candidats se réfèrent à leurs aînés [ağabeylerimiz], c'est-à-dire au mouvement des députés indépendants dans les années 1980 et 1990. Cette référence permet d'exprimer la méfiance de rigueur vis-à-vis des partis politiques grecs, soupçonnés de tout faire pour empêcher la 'minorité' d'élire « le nombre de représentants auquel elle a droit ».

De façon corollaire, le discours dominant au sein de l'arène publique «turque » fustige le système électoral et le barrage de $3 \%$ des voix à l'échelle nationale qui, depuis 1991, empêche à un parti «turc » comme le DEB de concourir aux élections législatives ${ }^{41}$, et plus généralement à tout candidat sans étiquette, laissant les candidats « turcs " «à la merci des partis grecs $»$. Le thème du leader charismatique ${ }^{42}$ est également très présent ; cela peut être illustré par les propos de Nazif Şakir, colistier d'İlhan Ahmet pour la Nouvelle Démocratie: "Je crois que toute société a besoin d'un leader. [...] Mon intention n'est que de combler un vide en attendant; je crois que d'ici un certain temps [la minorité] trouvera son leader naturel $\aleph^{43}$. Par ailleurs, se référant aux principes européens concernant les droits des minorités nationales, Rıza Kırlıökme appelle au rétablissement des collèges électoraux séparés des années 1920 et 1930, qui concernaient alors les juifs et les musulmans et permettaient à la " minorité » d'avoir jusqu'à quatre députés ${ }^{44}$. Enfin, le registre lexical et sémantique privilégié par les candidats «turcs» tout comme leurs promesses et propositions sont autant d'invocations de la Turquie : Burhan Baran, candidat du PASOK à Xanthi, déclare par exemple que « la Turquie doit aider les diplômés à trouver leur juste place au sein du champ politique en Thrace occidentale $»^{45}$.

\section{L'instauration d'un barrage électoral}

De 1989 à 1990, la Grèce a été marquée par une période d'instabilité gouvernementale liée à l'impossibilité pour le parti vainqueur de composer une majorité gouvernementale : trois élections successives ont eu lieu en juin et novembre 1989, puis en avril 1990. Les élections de juin 1989 furent annulées en raison de l'impossibilité pour la ND de dégager une majorité suffisante au Parlement. Le scénario se reproduisit en novembre 1989: il manquait un siège à la ND pour avoir la majorité absolue au Parlement. Or, "Du jour au lendemain [l'élu "turc" indépendant] İsmail Molla Rodoplu devint l'homme le plus en vue de l'actualité: de lui dépendait le prochain gouvernement" (Anastassiadou 1992: 77). La ND préféra finalement une éphémère coalition avec les 
communistes et de nouvelles élections furent d'emblée programmées. Mais le scénario se reproduisit en avril 1990: il manqua un siège la ND pour pouvoir constituer un gouvernement. Refusant de s'associer aux élus indépendants " turcs », elle se résigna à une fragile coalition avec un petit parti, le Parti du renouvellement démocratique ${ }^{46}$. C'est dans ce contexte que fut adoptée la nouvelle loi électorale : instaurant un barrage électoral de $3 \%$, cette mesure mettait de facto fin aux listes indépendantes en Thrace occidentale. Des listes furent tout de même constituées à Xanthi et dans le Rhodope en 1993, en signe de protestation, qui recueillirent de nombreux suffrages mais pour la première fois les musulmans ne furent pas représentés au Parlement grec.

26 La rhétorique en vigueur au sein de l'arène "turque " vise à masquer les rapports de force : en réalité ce sont les partis "grecs» qui choisissent leurs candidats «turcs». C'est aussi, du coup, une façon de minimiser le camouflet que constitue l'éventuelle défection d'un parti vis-à-vis d'un candidat, et les conflits ou tractations sous-jacentes. Une telle liberté de ton est en outre permise par l'usage de la langue turque ; c'est un discours à usage interne, sans risque de censure ou de polémique. Alors que l'usage de cette langue dans l'espace public était proscrit dans les années 1990, les autorités grecques ont dû en autoriser l'usage pour se conformer aux normes européennes; la langue turque est devenue un facteur essentiel de l'autonomisation de l'arène publique " turque » au sein du champ politique local. Par ailleurs, la relation inégalitaire entre les partis politiques et les candidats « turcs » est liée entre autres au faible engagement de ces derniers. Le déficit de compétences proprement politiques de la part des candidats «turcs » a été pointé au cours de la campagne dans un éditorial du journal Gündem ${ }^{47}$. Cette prise de position constitue une injonction contradictoire avec celle de décrier les partis ou de ne pas voter pour les "Grecs ", et résume en soi l'ambivalence de la campagne des candidats «turcs ». Car c'est le tribut à payer pour toute stratégie d'ascension sociale par une carrière politique : dénigrer le système grec dans lequel cette stratégie s'inscrit, sans lequel pourtant elle ne saurait se concrétiser.

\section{Le rôle du député au sein de la "minorité musulmane"}

27 Le député est avant tout l'intermédiaire entre les autorités grecques et la population musulmane, rurale à environ $80 \%{ }^{48}$, dont la frange la plus âgée est souvent illettrée, et surtout ignorante de la langue grecque. Dans une société de taille relativement restreinte comme celle-ci, le député connait souvent personnellement nombre de ses électeurs, et ceux-ci attendent de lui des services, mais aussi tout simplement qu'il se conforme aux codes sociaux liés à son statut social. L'idée de carrière politique est généralement associée à celle de carrière notabiliaire, au sens traditionnel du notable reconnu comme étant socialement supérieur, par hérédité mais aussi par des qualités humaines spécifiques qu'est censée lui procurer sa condition de notable. Galip Galip en est la parfaite illustration : héritier d'une dynastie locale, il était un novice en politique la première fois qu'il a été investi candidat par le PASOK en 1996, son seul capital politique était son nom. Le député est donc un notable, qui s'inscrit dans un rapport de patronage, mais aussi d'obligations réciproques avec ses électeurs ${ }^{49}$. C'est ainsi que malgré les efforts de sa femme pour le représenter dans les villages (voir infra), le député sortant du Rhodope Galip Galip (PASOK) s'est vu reprocher par ses administrés dans les villages de ne pas leur avoir suffisamment rendu visite au cours de son mandat et de sa campagne ${ }^{50}$. Le rôle social de ces visites du notable dans les villages est tel qu'il est investi aussi par les candidats "grecs », ce qui a conduit le président du Conseil 
consultatif, à mettre en garde les électeurs musulmans contre ces "personnes qui viennent à votre porte quatre fois pour avoir votre précieux vote, mais qui pendant les quatre prochaines années ne passeront peut-être même pas dans votre village prendre de vos nouvelles $»^{51}$, dont on comprend qu'elles sont " grecques » puisque étrangères aux coutumes et savoir-vivre « turcs».

On attend aussi du député qu'il soit présent aux cérémonies de mariage ou de circoncision, du moins dans les familles importantes, qui marquent ainsi leur prestige social. Si le député est supposé rendre visite à ses administrés, ceux-ci attendent aussi qu'il leur rende des services. D'ailleurs, une grande partie du temps de l'élu est consacré à recevoir ses électeurs et administrés, à écouter leurs doléances, à enregistrer leur demande d'emploi, etc. Le député est l'interlocuteur pour régler des problèmes administratifs aussi bien que pour trouver un emploi. C'est ce que montre une interview accordée par İlhan Ahmet au journal Gündem quelques mois après son élection, dans laquelle il détaille son emploi du temps hebdomadaire : "Je passe quatre jours par semaine à Athènes. [...] Je rentre à Komotini le jeudi soir très tard. Le vendredi, je m'entretiens en moyenne avec 50 personnes dans mon bureau. Les jours où je suis ici je rencontre mes concitoyens et je visite les villages $\|^{52}$. C'est aussi la façon dont son prédécesseur, Galip Galip, nous avait décrit ses fonctions : "Vous savez moi je fais un travail d'administration ici. Les personnes de la minorité souvent ne parlent pas le grec. Pour eux mon bureau de député sert à faire le lien avec la mairie, la préfecture. Le moindre permis, la moindre fiche d'état civil ou déclaration officielle, tout ce qui doit passer par l'administration passe par nous. Je dois recevoir tout le monde. Mon travail de député c'est beaucoup ça aussi. Vous avez vu le petit vieux là tout à l'heure? Il venait prendre conseil pour une histoire de permis de construire. Nous faisons tout cela ici $»^{53}$.

\section{Les registres d'action}

\section{La place prépondérante des villages dans la campagne électorale}

Les villages des départements de Xanthi et du Rhodope sont le théâtre de la majeure partie de la campagne électorale, pour les candidats " grecs » comme «turcs » ${ }^{54}$, alors qu'ils sont très peu intégrés à l'espace social et économique urbain. Il y a à cela deux raisons principales : la première tient à la loi électorale grecque, qui limite à un seul par parti les meetings en ville, la seconde est liée au rôle du notable décrit ci-dessus et à ce qu'on pourrait appeler les rituels politiques locaux. La plupart des meetings ont lieu dans les villages, et se transforment éventuellement en réunions électorales en petits comités. Ils se tiennent pour les hommes dans les villages, aussi bien au café qu'à la mosquée ${ }^{55}$; le rituel politique intègre en effet le rite religieux et il est de bon ton pour les candidats 'turcs' d'être vus au moins une fois à la prière du vendredi, quand bien même ils ne fréquentent pas la mosquée par ailleurs. Par ailleurs les discours religieux et sermons, notamment ceux des muftis dissidents, constituent souvent l'occasion d'exprimer une opinion politique ${ }^{56}$. Les femmes quant à elles vont de maison en maison, jouant des codes de sociabilité pour promouvoir la candidature de leur mari ou parent. La femme de Galip Galip, déjà six mois avant les élections, faisait des visites de courtoisie dans les villages des alentours de Komotini, sous un prétexte ou un autre, les bras chargés de pâtisseries, félicitant telle jeune mariée, encourageant les uns et les autres pendant l'enfilage du tabac, et prenant accessoirement des renseignements sur «ceux de la droite " $^{57}$. Au lendemain des élections, les observateurs locaux ont mis en 
parallèle l'incroyable travail de terrain d'İlhan Ahmet, qui a sillonné le Rhodope en visitant chaque village au moins une fois, souvent deux, avec le fait que Galip Galip ait attendu les dernières semaines pour se mettre en campagne. Ce fut notamment le commentaire de la rédactrice en chef du journal Gündem, la veille du scrutin, alors que la victoire d'İlhan Ahmet était anticipée : «Et bien ça veut dire qu'il y a un sacré travail derrière, et que c'est payant $"^{58}$. D'autant que les candidats (ou plutôt leur «convoi ») sillonnent rarement les villages les mains vides.

À quelques jours du scrutin, un reportage du quotidien de Turquie Milliyet relatait les tournées dans les villages des candidats "turcs" et mentionnait les «menus objets, kurabiye [gâteaux secs recouverts de sucre glace], dentifrice et chocolats » que ceux-ci offrent à la ronde ${ }^{59}$. La rédactrice en chef de Gündem se montra incrédule quant à ses informations, qui la firent rire de bon cœur. En revanche, selon elle, les candidats se rendent d'abord chez l'imam du village et lui demandent la liste des naissances, mariages, et circoncisions récents, puis ils vont rendre visite aux familles concernées avec des cadeaux ${ }^{60}$. Un autre aspect, et non des moindres, de la campagne dans les villages concerne la distribution des bulletins de vote. C'est en effet dans les villages, plus particulièrement dans les villages non mixtes, qu'on trouve le plus de personnes ne sachant pas le grec, toutes générations confondues. En Thrace occidentale est tolérée la distribution anticipée de bulletin, en plus de ceux qui se trouvent dans les bureaux de vote le jour du scrutin, ce qui est interdit dans toute autre région de Grèce. Cela permet aux candidats «turcs» de distribuer à l'avance des bulletins de vote où leur nom est déjà coché sur la liste de leur parti ; ils font également imprimer leur photo sur l'enveloppe afin d'éviter toute confusion de la part des électeurs analphabètes, ou simplement qui ne lisent pas le grec ${ }^{61}$. Pour ces élections, en tout 120000 bulletins ont été imprimés à l'initiative des différents candidats ${ }^{62}$, soit un chiffre bien plus élevé que le nombre d'électeurs réels (voir les chiffres en annexe). Les membres des équipes de campagne distribuaient eux-mêmes ces bulletins dans les villages ou à l'arrivée des bus venant de Turquie. Lors du voyage en bus d'Istanbul à Komotini, l'avant-veille des élections, des membres de l'équipe de campagne de Mehmet Ahmet sont montées dans le bus peu après la frontière, près d'Alexandroupolis (Dedeağaç) pour distribuer des tracts et bulletins de vote. Environ une heure plus tard, lorsque le bus s'arrêta à Şapça (Sapes), à une trentaine de kilomètres de Komotini, une autre équipe est montée pour distribuer les bulletins de vote et la brochure de Galip Galip.

\section{La campagne électorale parallèle des principaux candidats « turcs » et le recours symbolique à la Turquie}

31 L'existence d'une arène "turque " autonome au sein du champ politique local est attestée aussi par le fait que les candidats « turcs » mènent leur campagne en parallèle de celle du parti qu'ils représentent, en turc et autour d'enjeux spécifiques. Symboliquement, les deux principaux candidats « turcs » du PASOK et de la ND avaient un siège de campagne distinct de celui de leur parti : Galip Galip avait installé le sien dans son bureau de député, avec une annexe dans le cabinet dentaire de sa femme, situé dans une rue adjacente. Quant à İlhan Ahmet, il était fier d'avoir loué comme siège de campagne le bureau qui avait déjà servi à cet usage à Sadık Ahmet. Les principaux candidats «turcs » recouraient à des conférences de presse, qui étaient en fait souvent des dîners offerts aux seuls journalistes des médias turcophones locaux et 
de Turquie ${ }^{63}$. Mais le plus frappant est le jeu discursif auquel sont tenus les candidats du PASOK et de la ND pour se conformer au discours en vigueur au sein de l'arène " turque ", et la liberté totale dont ils bénéficient pour cela. Les candidats minimisent leur affiliation partisane, dénigrant même à l'occasion le parti pour lequel ils sont candidats ; c'est certainement İlhan Ahmet qui est allé le plus loin dans cette voie. Lors de l'un de ses derniers meetings de campagne, il tint les propos suivants : «Pendant la période 1985-95, la minorité a compris que PASOK et ND c'est la même chose, et a donné 30000 voix à la liste des candidats menée par feu Sadık Ahmet». De façon classique, il reprit le thème selon lequel, depuis les années 1990 et l'instauration d'un barrage électoral, «la minorité est obligée de jouer le jeu des partis». Surtout, il fit preuve d'une certaine agressivité en utilisant le terme çorbact ${ }^{64}$, à connotation péjorative, pour se référer aux «Grecs » qui ont décidé de « diviser la minorité ». Il fut le seul candidat à mentionner la crise de Chypre au cours de cette campagne, celle-ci ayant pourtant surdéterminé la vie politique locale dans les années 1980. Il utilisa pour cela le terme officiel en Turquie, barış harekatı ou "opération de paix", alors qu'en Grèce comme dans l'Union européenne on parle « d'occupation du nord de Chypre " par l'armée turque ${ }^{65}$.

Un tel discours n'est peut-être pas sans rapport avec le fait que, le scrutin approchant, le consulat de Turquie et le Conseil consultatif avaient changé de stratégie à l'égard de sa candidature. Dans les derniers jours de la campagne, İlhan Ahmet a été contacté par le consul général de Turquie, qui lui a demandé de poser sur une photo aux côtés de Galip Galip, son rival du PASOK ${ }^{66}$. La photo a été publiée en couverture de Gündem l'avant-veille du scrutin: on voit les deux candidats en train de se serrer les mains; juste en dessous une autre photo montre les deux candidats de Xanthi pour le PASOK et la ND, Burhan Baran et Türkeş Hacımemiş, côte à côte et souriants ${ }^{67}$. Il s'agissait de montrer l'unité des candidats "turcs » à la veille des élections. Selon İlhan Ahmet, c'était aussi une façon pour le consulat général de Turquie et le Conseil consultatif, à la veille de la victoire de la Nouvelle Démocratie ${ }^{68}$, d'assurer un parrainage de dernière minute pour le candidat du parti de la future majorité. Celui-ci dit avoir accepté de jouer le jeu, dans la mesure où il s'agissait de préparer l'après-élection : prendre en compte la nécessité de composer avec ces acteurs incontournables en Thrace ${ }^{69}$. Quant à Galip Galip, le discours qu'il a tenu lors de son dernier meeting de campagne, auquel nous avons assisté le 5 mars 2004 dans un village des environs de Komotini, constituait un exercice difficile : il condensait les thèmes obligatoires pour les candidats 'turcs' tout en y associant le PASOK, mais en ne manquant pas de se distancier des "Grecs", éventuellement en employant des termes à connotation péjorative. Il rendit néanmoins hommage aux candidats "grecs » de son parti qui, dans les villages, «se sont comportés en gentlemen » en invitant les "Turcs à voter pour leurs propres candidats». Cela en dit long sur la façon dont l'arène publique "turque " impose ses cadres rhétoriques à l'ensemble des acteurs du champ politique local.

La Turquie se manifeste dans la campagne en tant que réseau d'institutions et d'acteurs étatiques, on l'a vu, mais aussi en tant que référent politique et symbolique. De façon anecdotique mais significative, İlhan Ahmet s'est inspiré de l'initiative de Cem Uzan (leader du Genç Parti, le Parti jeune) lors des élections législatives turques de novembre 2002 (Massicard 2003) : il a fait envoyer par l'un de ses parents résidant en Turquie un SMS à toutes les personnes dont il possédait le numéro de téléphone mobile, qui disait "la Turquie soutient İlhan Ahmet». Enfin, la Turquie a pris une part active dans la campagne et le déroulement des élections par l'intermédiaire des milliers de membres 
de la «minorité musulmane » résidant en Turquie qui ont franchi la frontière pour se rendre aux urnes.

\section{La mobilisation des électeurs « turcs » de Turquie et d'Allemagne} " grecs » et «turcs », mais aussi des autorités turques par l'entremise des associations de Turcs de Thrace de Turquie, à l'électorat constitué par les membres de la minorité musulmane résidant en Turquie ${ }^{70}$. En 2002, pour la première fois les candidats " grecs » aux élections locales ont pris en compte cet électorat et effectué pour certains un séjour en Turquie au cours de leurs tournées électorales ${ }^{71}$. Quelques jours après les élections municipales et préfectorales, la presse de Turquie relatait en outre l'information suivante : plusieurs candidats grecs, notamment Aris Yannakidis (en tête de la liste soutenue par le PASOK dans le Rhodope), avaient affrété dix bus pour faire venir les électeurs d'Istanbul ${ }^{72}$. Le journal Gündem n'a pas relayé cette information, probablement en raison du discours dominant au sein de l'arène "turque ", stigmatisant la coopération avec les candidats " grecs », ou en raison de la concurrence entre partis politiques grecs et associations de Turcs de Thrace (en Thrace et en Turquie) pour tirer le bénéfice de la mobilisation «turque ». Ainsi, alors que lors des élections législatives de 1990 et 1993 les bus venus de Turquie avaient été bloqués à la frontière, et finalement arrivés après la fermeture des bureaux de vote, désormais acteurs politiques 'grecs' et 'turcs' rivalisent d'attentions vis-à-vis de l'électorat musulman gurbetçi [exilé].

L'électorat «turc de Thrace » en Turquie, estimé à 10000 personnes environ, était en mesure comme on l'a vu de faire pencher sensiblement la balance pour l'un ou l'autre parti puisque l'écart entre le PASOK et la ND s'annonçait très faible ${ }^{73}$, et à ce titre était courtisé par les partis « grecs» tout autant que par les candidats «turcs ». De fait, la pratique inaugurée en 2002 a pris en 2004 de tout autres proportions afin de faire venir gratuitement de Turquie le plus d'électeurs possible. Concurremment avec les partis politiques grecs, les associations de Turcs de Thrace en Turquie ont mobilisé leurs "pays" [hemşehri] (Hersant 2005b). La mobilisation a consisté à convaincre les personnes ayant conservé la nationalité grecque d'aller voter, mais aussi à prévoir l'organisation logistique de leur transport. En février 2004, le président et plusieurs des dirigeants de l'Association de Solidarité des Turcs de Thrace occidentale ${ }^{74}$ ont organisé des réunions dans chaque association de quartier d'Istanbul, arguant de l'inquiétude de la «mère-patrie » quant à la possibilité qu'iln'y ait pas de représentant turc au Parlement grec $^{75}$. Hormis ces arguments, plusieurs de nos interlocuteurs ont mentionné une rumeur à laquelle ils croyaient fermement : ceux qui n'iraient pas voter subiraient ensuite des mesures de rétorsion de la part des autorités grecques, par exemple au moment de faire renouveler leur passeport ${ }^{76}$. Dans les semaines qui ont suivi, chaque antenne de l'Association de Solidarité a été chargée d'inscrire les électeurs souhaitant faire le voyage. Au final, une centaine de bus a pris le départ de plusieurs grandes villes de Turquie (Ankara, İstanbul, Bursa, İzmir, Soma, Edremit, Yalova, Adapazarı, Kocaeli) ${ }^{77}$ à destination d'Alexandroupolis, Komotini, Sapes, Xanthi, pour un coût qui peut être estimé à 50000 euros $^{78}$. Environ 8000 personnes ont franchi la frontière ${ }^{79}$, ce qui laisse supposer que le nombre de "Turcs» de Thrace occidentale résidant en Turquie tout en ayant la nationalité grecque est bien supérieur, puisque tous n'ont 
évidemment pas fait le voyage ; les dirigeants associatifs eux-mêmes ne pensaient pas que ce public était si nombreux ${ }^{80}$.

La Fédération européenne des Turcs de Thrace occidentale ${ }^{81}$, rassemblant essentiellement des migrants installés en Allemagne, n'a pas eu les moyens de mettre en œuvre seule cette mobilisation. Bien qu'il nous ait été impossible d'obtenir des informations précises à ce sujet en raison d'une pratique de la « langue de bois » parmi les acteurs associatifs interrogés, il semble que la Fédération ait organisé le transport de ces électeurs depuis l'Allemagne conjointement avec les représentations locales du PASOK et de la $\mathrm{ND}^{82}$. Les partis politiques grecs mobilisent de fortes sommes pour affréter des avions, notamment depuis l'Allemagne, et permettre aux ressortissants grecs de faire le voyage à bas prix (moins de 100 euros aller et retour), sans avoir auparavant cherché réellement à atteindre les musulmans de Thrace. Environ 600 électeurs sont venus d'Allemagne, ce qui était inédit: les musulmans de Thrace qui y vivent ne participent jamais, d'habitude, aux élections ${ }^{83}$. Étant donné les efforts de mobilisation mis en œuvre en Turquie, étant donné la longueur du voyage (de six à huit heures en bus depuis Istanbul, plus depuis Bursa ou Ankara), il est raisonnable de considérer comme hypothèse le fait que les électeurs qui franchissent la frontière le font pour apporter leur voix aux candidats turcs quel que soit le scrutin ${ }^{84}$; tandis que les déterminants du vote autochtone répondent à des enjeux spécifiques, qui varient en fonction du caractère local ou national du scrutin. On va voir dans un premier temps en quoi l'hypothèse d'une 'fuite' massive des votes musulmans vers les candidats " grecs » ne permet pas d'expliquer la sous représentation de la minorité aux élections législatives de 2004.

\section{L'échec relatif de la mobilisation communautaire : au-delà des apparences}

\section{L'impéritie de l'argument « ethnique »}

Durant la campagne et à peine les résultats connus, tous les acteurs politiques et commentateurs «turcs» ont unanimement accusé la défection des électeurs musulmans qui auraient voté massivement pour des candidats "grecs». Nous avons trouvé dans la presse turque et turcophone locale des estimations de ce phénomène : lors du scrutin de 2000, par exemple, de 55 à $60 \%$ des votes musulmans seraient allés à des candidats «chrétiens $»^{85}$. Seulement, ces chiffres ne sont jamais justifiés, et la source n'en est jamais mentionnée. Implicitement, ils expliquent d'autre part l'absence de député "turc» dans le département de Xanthi depuis 1996 par la forte concentration de locuteurs du pomaque, un dialecte du bulgare ${ }^{86}$ : les Pomaques seraient moins « turcs » car bulgarophones, donc plus portés à voter pour les candidats " chrétiens ». Or, une telle assertion ne résiste pas à l'étude méthodique des résultats. L'analyse détaillée des résultats montre en premier lieu que la déperdition de voix pour les candidats « turcs » est en fait plus importante dans le département du Rhodope que dans celui de Xanthi. Dans les deux départements, l'appel au vote communautaire a bien fonctionné, si l'on en juge par les chiffres obtenus en comparaison des élections précédentes. Mais en proportion, c'est dans le département de Xanthi que la mobilisation en faveur du vote « turc " a été le plus suivie, alors que la tendance inverse s'observe dans le Rhodope, malgré l'augmentation en valeur absolue des votes pour les 
candidats «turcs », largement inférieure à l'augmentation du nombre de suffrages musulmans exprimés.

Le vote musulman dans les départements de Xanthi et du Rhodope (élections de 1996, 2000 et 2004)

\begin{tabular}{|c|c|c|c|}
\hline XANTHI & 1996 & 2000 & 2004 \\
\hline \multicolumn{4}{|l|}{ Échelon départemental } \\
\hline Estimation des votes musulmans exprimés & 25300 & 26500 & 28500 \\
\hline Votes totalisés par les candidats « turcs » & 14741 & 13097 & 17147 \\
\hline \multicolumn{4}{|l|}{ Proportion des votes } \\
\hline pour les candidats « turcs » & $58,26 \%$ & $49,42 \%$ & $60,16 \%$ \\
\hline \multicolumn{4}{|l|}{ Échantillon représentatif } \\
\hline Votes musulmans exprimés & 12496 & 12638 & 13753 \\
\hline Votes totalisés par les candidats « turcs » & 7499 & 6024 & 5239 \\
\hline \multicolumn{4}{|l|}{ Proportion de votes } \\
\hline pour les candidats « turcs » & $60,01 \%$ & $47,67 \%$ & $38,09 \%$ \\
\hline RHODOPE & 1996 & 2000 & 2004 \\
\hline \multicolumn{4}{|l|}{ Échelon départemental } \\
\hline Estimation des votes musulmans exprimés & 35000 & 35000 & 42800 \\
\hline Votes totalisés par les candidats « turcs » & 27833 & 28905 & 32553 \\
\hline \multicolumn{4}{|l|}{ Proportion de votes } \\
\hline pour les candidats « turcs» & $79,52 \%$ & $82,59 \%$ & $76,06 \%$ \\
\hline Votes musulmans exprimés & 14005 & 14509 & 16033 \\
\hline Votes totalisés par les candidats « turcs » & 10290 & 11902 & 13670 \\
\hline \multicolumn{4}{|l|}{ Proportion de votes } \\
\hline pour les candidats « turcs » & $73,47 \%$ & $82,03 \%$ & $85,26 \%$ \\
\hline
\end{tabular}

Tout d'abord, le nombre de votes exprimés (et valides) a augmenté en 2004 de près de 10000 dans chacun des deux départements du Rhodope et de Xanthi par rapport aux élections législatives de 1996 et $2000^{87}$. Les résultats des trois dernières élections font apparaître, dans les deux départements de Xanthi et du Rhodope, des disparités dans la discipline de vote pour les candidats "turcs». Si les électeurs "musulmans» sont 
moins nombreux à Xanthi ; derrière cette réalité numérique, on constate qu'ils votent différemment de ceux du Rhodope. La proportion de votes musulmans pour les candidats « turcs » dans le département de Xanthi $(60,16 \%)$ a été supérieure à celle de 1996 (58,26\%), année ou trois députés «turcs » avaient été élus. Dans le Rhodope en revanche, c'est l'inverse (79,52\% en 1996, 76,06 \% en 2004); cette proportion est même nettement inférieure à ce qu'elle était en 2000 (82,59\%). Les candidats "turcs » ont pourtant totalisé 32533 voix sur les 42800 votes musulmans exprimés dans le Rhodope ${ }^{88}$, soit bien plus en valeur absolue que lors des scrutins précédents, probablement grâce au nombre d'électeurs venus de l'étranger. Une autre différence intervient au niveau des échantillons : alors que l'échantillon du Rhodope présente le même comportement de vote que celui observé à l'échelle départementale, l'échantillon de Xanthi montre une évolution inverse. Dans les deux départements le nombre de votes pour les candidats «turcs» augmente entre 1996 et 2004, mais il décroît fortement sur la même période dans l'échantillon de Xanthi, alors que le nombre de voix exprimées dans cette circonscription est en constante augmentation: la proportion de votes pour les candidats « turcs » y est passée de $60 \%$ à 38 \% quand, au niveau départemental elle est toujours de $60 \%$. L'explication de la faible représentation des musulmans au Parlement est en fait liée à des facteurs différents dans les deux départements, du moins tels que les résultats apparaissent au sein des deux échantillons constitués.

\section{L'importance des bulletins non nominatifs à Xanthi fausse le jeu de la répartition des sièges}

39 Lorsque l'on regarde en détail les résultats concernant l'échantillon de Xanthi (voir en annexe), on voit que ce sont les candidats «turcs » qui ont totalisé le plus grand nombre de voix au sein de leur parti, lors des élections de 1996, 2000 et 2004. En 2000, seul le candidat de DIKKI, Mustafa Köşe, est distancé par l'un de ses colistiers " grecs » dans la municipalité de Mikis. En 2004, hormis Türkeş Hacımemiş (ND), qui est distancé par son colistier Alexandros Kondos dans la municipalité de Mikis ${ }^{89}$, et Sebahaddin Tosunoğlu (DIKKI) qui ne recueille que quatre voix sur l'ensemble de l'échantillon ${ }^{90}$, les candidats "turcs» recueillent la majorité des voix de leur parti dans chaque circonscription, voire la quasi-totalité dans le cas des candidats du Synaspismos et du KKE. En 2004, Burhan Baran (PASOK) notamment est largement en tête au sein de sa liste dans tous les villages, même si ses colistiers totalisent un nombre de voix non négligeable. Or, malgré cet écart en valeur absolue, sur l'ensemble de l'échantillon les candidats « grecs » obtiennent $31,34 \%$ des voix accordées aux cinq principaux partis en lice, contre $38,75 \%$ pour les candidats «turcs ». Pourtant, si l'on y regarde de plus près on remarque qu'à l'échelle de l'échantillon de Xanthi, la proportion de votes pour les candidats "turcs" par rapport au total des votes exprimés baisse plus vite sur la période que le nombre de votes pour les candidats « turcs » en valeur absolue.

En effet, par rapport aux élections de 2000, le nombre de voix pour les candidats "grecs» et pour les candidats "turcs» a diminué, alors que le nombre de voix exprimées dans l'échantillon a augmenté de plus de 1000 entre 2000 et 2004, passant de 12638 à 13 753. Pour comparaison, de 1996 à 2000, alors que le nombre de votes exprimés dans l'échantillon était resté stable (12 496 en 1996, 12638 en 2000), le nombre de votes donnés aux candidats " grecs" avait augmenté de 3531 à 5550 . Dès 
lors, pour comprendre pourquoi l'augmentation considérable du nombre de votes exprimés entre 2000 et 2004 (qui représentent environ $12 \%$ du total des votes exprimés) ne se retrouve pas dans les votes pour les candidats «turcs» ni pour les candidats "grecs", il faut prendre en considération une autre variable que le pourcentage de votes totalisés par les candidats "turcs", à savoir les modalités particulières du scrutin de liste en Grèce et la possibilité de voter pour un parti sans désigner de candidat.

\section{Le scrutin de liste en Grèce ${ }^{91}$}

41 Les élections législatives en Grèce se font, depuis 1993, selon un scrutin de liste à un seul tour. Les électeurs votent pour la liste d'un parti et peuvent en outre exprimer (ou non) leur préférence pour un candidat en apposant une crois devant son nom, indépendamment du rang de ce candidat sur la liste (Hlepas 2003). De ce fait, les candidats d'une même liste (quatre pour les grands partis) dans chaque circonscription sont en concurrence entre eux de façon beaucoup plus nette que pour les élections locales, qui se déroulent selon un scrutin de liste classique à deux tours. Ce système assure au parti qui remporte les élections la majorité absolue des sièges au Parlement, quelle que soit l'avance avec laquelle il a obtenu la majorité dans les urnes; il permet également, par la dose de scrutin proportionnel qu'il comporte, aux petits partis de siéger au Parlement, dès lors qu'ils franchissent le barrage de $3 \%$ des voix à l'échelle nationale.

Cette variable n'est jamais prise en compte par les commentateurs «turcs " locaux. Rıza Kırlıdökme qui, rappelons-le, souhaitait le rétablissement de collèges électoraux séparés, consacra ses éditoriaux pendant huit semaines à l'analyse des résultats électoraux pour dénoncer la 'faute' des électeurs de notre échantillon ${ }^{92}$. Il se contenta de soustraire du nombre de votes exprimés le nombre de votes totalisés par les candidats « turcs » de chaque parti pour en déduire de façon automatique le nombre de votes accordés à des candidats "grecs». Or, la particularité de notre échantillon de Xanthi, par rapport à celui du Rhodope, est l'impressionnant écart entre le nombre de bulletins nominatifs et le total des voix enregistrées par chaque parti. Dès 1996, cet écart était en moyenne plus élevé dans cet échantillon que dans celui du Rhodope, mais le phénomène s'est accentué en 2004 : le taux de bulletins non nominatifs a atteint alors $29,90 \%$ du nombre de bulletins pour les cinq principaux partis en compétition. Si l'on regarde de plus près, on s'aperçoit d'ailleurs que ce sont le PASOK et la ND qui totalisent le plus grand nombre de bulletins non nominatifs, mais uniquement à Mikis. Le nombre de bulletins non nominatifs pour le PASOK (1739) y est supérieur au nombre de votes exprimés en faveur de Burhan Baran (1 664). Dans la même municipalité, le nombre de bulletins non nominatifs pour la Nouvelle Démocratie (2 034) est plus de quatre fois supérieur au nombre de voix totalisées par Türkeş Hacımemiş (492). C'est ce paramètre qui semble tout changer dans le calcul de la répartition des sièges; en effet, en 1996, la proportion identique de voix attribuées à des candidats "grecs» $(30 \%)$ n'a pas empêché l'élection d'un député «turc » à Xanthi. En 2004, la proportion de votes " grecs » a même nettement baissé par rapport à ce qu'elle était en 2000 (31,34 \% contre $44,70 \%)$. Ces deux variables conjuguées expliquent la baisse du vote pour les candidats "turcs ", tant en valeur absolue qu'en pourcentage ; on peut néanmoins relever au niveau micro-local la spécificité de la municipalité de Mikis, la plus importante et la seule où apparaisse clairement une baisse des votes pour les candidats « turcs ${ }^{93}$. 

tendance inverse à celle de l'ensemble de l'électorat musulman dans le département de Xanthi et que, enfin, cet échantillon représente environ la moitié de l'électorat musulman estimé du département, on peut envisager que c'est le phénomène observé dans notre échantillon qui a empêché l'élection d'un député "turc» dans le département. Le reste de l'électorat musulman étant localisé dans la ville de Xanthi et dans les zones villageoises périurbaines, on peut émettre l'hypothèse d'un comportement électoral différencié entre la ville et la zone montagneuse de notre échantillon. Nous ne disposons pas d'éléments permettant d'expliquer ce phénomène massif de vote non nominatif, observable uniquement pour les élections de 2004 ; cela n'a pas de rapport en tout cas avec la position des candidats « turcs » sur la liste (voir l'annexe sur le mode de scrutin). On peut simplement rappeler le rôle des villages qui constituent notre échantillon dans la lutte symbolique que mènent "Turcs» et «Grecs » pour prouver ou au contraire minimiser l'identité turque de la minorité. Les habitants de cette circonscription proche de la frontière bulgare sont tous, ne serait-ce que dans la sphère privée, locuteurs du pomaque (dialecte du bulgare), quelle que soit par ailleurs leur identité politique. Le nombre important de bulletins non nominatifs peut éventuellement être lu, dans cette perspective, comme un refus ou une impossibilité à prendre position parmi ces injonctions contraires. Quoi qu'il en soit, l'omission de cette variable liée au mode de scrutin a permis aux acteurs du mouvement identitaire " turc', en d'autres circonstances, de gonfler artificiellement le score des candidats « turcs », notamment celui de Sadık Ahmet en 1989 (Hersant 2007).

\section{La dispersion des voix entre les candidats «turcs » dans le Rhodope}

Précisons qu'à la différence de notre échantillon de Xanthi, celui du Rhodope fait apparaître une nette augmentation de la proportion de votes «turcs", alors qu'elle a baissé dans l'ensemble du département - en dépit de l'augmentation significative du nombre d'électeurs musulmans et du nombre total d'électeurs par rapport aux scrutins de 1996 et 2000. Pour les élections de 2004, il y a près de dix points de différence entre les deux échelles observées (tableau ci-dessus). On ne peut de ce fait exclure à l'échelle $\mathrm{du}$ département (et a fortiori en zone urbaine) le maintien d'un taux de votes relativement élevé pour les candidats " grecs ", ou encore l'hypothèse d'un vote non nominatif important, comme dans le premier échantillon. De plus, dans ce second échantillon la discipline de vote communautaire, qui implique obligatoirement un vote nominatif, a été de ce point de vue, mieux respectée. En 1996, on l'a vu, la proportion de bulletin non nominatifs par rapport au nombre de voix pour les partis présentant des candidats «turcs » était assez proche dans nos deux échantillons : 6,27 \% à Xanthi et 4,97 \% dans le Rhodope. Cette proportion n'a ensuite cessé de décroître dans le second échantillon, passant à $4,65 \%$ en 2000 et à $2,88 \%$ en 2004, présentant en cela une évolution inverse à celle du premier échantillon. Pour autant, le nombre d'élus n'augmente pas, il est même en baisse depuis 1996. Or, on observe que le scrutin où le Rhodope a envoyé deux députés "turcs " au Parlement est également celui où la proportion de votes pour des candidats «grecs " a été la plus élevée. En 1996, 18,54 \% des votes pour les partis présentant au moins un candidat "turc " étaient destinés à l'un des colistiers " grecs », et non pas « turcs ». En 2000 cette proportion tombe à 11,98 $\%$, mais un seul député « turc » est élu; idem en 2004 où la proportion de votes pour les 
candidats « grecs » est de 11,30 \% (mais en valeur absolue ils sont plus nombreux qu'en 2000).

Proportionnellement, les votes pour les candidats "grecs » ont baissé entre 2000 et 2004 de façon bien moins significative que dans le département de Xanthi, et cette décrue du nombre de votes pour les candidats "grecs" ne suffit pas à faire élire plus d'un député "turc»; il faut donc envisager ici la dispersion des votes comme facteur de sous représentation de la « minorité musulmane » au Parlement grec. L'élection d'un seul député « turc » dans le Rhodope, malgré la forte mobilisation des électeurs, semble ici trouver ses raisons principalement dans la dispersion des voix entre tous les candidats " turcs » : il y a une double dispersion des votes musulmans, à la fois entre partis et entre candidats "turcs" d'un même parti. En effet, en raison des règles régissant la compétition politique et le mode de scrutin, le jeu de la concurrence entre candidats d'un même parti est tout aussi présent entre candidats «turcs " qu'entre candidats "grecs» et "turcs ». En outre, en 1996 lorsque Galip Galip et Mustafa Mustafa ont été élus, il n'y avait que sept candidats «turcs » dans le Rhodope. Il y en avait douze en 2000 et dix en 2004 ; depuis 2000 , même le Synaspismos présente deux candidats « turcs».

\section{Déficit de capital partisan et d'expérience de la gestion des affaires publiques par rapport aux candidats « grecs »}

Hormis la dispersion des voix, la différence de profil entre les candidats "grecs » et "turcs" semble déterminante pour expliquer que très peu de candidats "turcs " parviennent à fédérer suffisamment de votes pour être élus : aucune personnalité "turque " ne se détache suffisamment par son expérience politique de gestion des affaires publiques. Les élus « turcs » à la députation ont pour point commun avec leurs rivaux "grecs" d'être des notables locaux (par héritage ou par carrière), et de maitriser aussi bien les codes politiques et sociaux « grecs » que « turcs »; en outre leur trajectoire familiale, scolaire et professionnelle les a conduits à être parfaitement bilingues. Pour la première fois en 1996, les trois députés «turcs » au Parlement grec étaient titulaires d'un diplôme de l'enseignement supérieur. Il y a de ce point de vue une incontestable homogénéisation des profils sociologiques des candidats à la députation en Thrace occidentale - si ce n'est qu'il ne se trouve de candidates et a fortiori de députées que parmi les "Grecs »; le diplôme universitaire associé à une profession socialement prestigieuse tend à remplacer l'hérédité dans la qualification du notable. Galip Galip (architecte), İlhan Ahmet (avocat) et Burhan Baran (chirurgien) sont les trois candidats "turcs" qui ont totalisé le plus de voix aux élections législatives de 2004, les deux premiers dans les Rhodope, le troisième à Xanthi sur la liste du PASOK. Le cas de Burhan Baran est plutôt exceptionnel puisqu'il a passé près de vingt ans à l'étranger (Turquie et Allemagne). Hormis son brillant parcours universitaire, le fait qu'il ait acquis durant sa scolarité une bonne maîtrise de la langue grecque n'est certainement pas étranger à sa reconversion politique réussie ${ }^{94}$. Mais si l'on opère une comparaison avec les colistiers « grecs » d'İlhan Ahmet et Galip Galip qui ont été élus dans le Rhodope (voir les biographies en annexe), le déficit d'expérience au sein d'un appareil de parti et dans la gestion des affaires publiques des deux personnalités "turques" apparait clairement. De ces trois candidats, seul Burhan Baran avait été élu lors des élections locales de 2002, à l'assemblée départementale de Xanthi ; İlhan Ahmet avait fait un piètre score, quant à Galip Galip, il est le seul 
candidat " turc » à la députation à n'avoir jamais brigué de mandat local (à la différence d'Ahmet Mehmet et Mustafa Mustafa qui, eux, bénéficient d'un capital militant acquis de longue date au sein de leur parti). Or, de plus en plus, les candidats «turcs » aux élections nationales doivent avoir une expérience reconnue au sein du système «grec ».

\section{Conclusion}

Cette étude montre l'intérêt des outils d'analyse de la sociologie politique pour appréhender des configurations politiques et sociales surdéterminées par une grille d'analyse identitaire et ethnique. En dépit du discours de la «minorité opprimée », la disparition de toute action protestataire depuis les années 1990 illustre a contrario la légitimité et la visibilité des revendications «turques» dans le champ politique local. En outre, le phénomène observé de sous représentation électorale des "musulmans » semble être un phénomène de long terme qui révèle une intégration croissante au système politique grec, d'autant que celui-ci s'est largement ouvert depuis les années 1990, permettant à de nombreux musulmans de participer au jeu politique en se déclarant candidats aux élections législatives. Là est le paradoxe: désormais la présence d'un ou plusieurs musulmans sur les listes de partis politiques est considéré comme un atout électoral, or c'est justement cette pléthore de candidatures qui obère la représentation de la minorité au Parlement. Du coup, si les revendications liées à la «turcité » restent centrales dans la rhétorique politique locale, c'est justement cette centralité qui leur fait perdre une partie de leur charge mobilisatrice en contexte électoral. En effet, l'inefficacité du vote "communautaire » en 2004 n'est finalement que peu liée à un défaut de mobilisation, comme le montre la forte augmentation des votes exprimés dans les deux départements; de façon corollaire le vote «turc » est manifestement porté en grande partie par les électeurs venus de Turquie. On note en outre une émancipation du vote par rapport à la ligne électorale donnée par le consulat général de Turquie ${ }^{95}$. Les différentes rivalités, pressions et marchandages de voix sont suffisamment complexes pour que, alors même que les candidats " grecs " reprennent à leur compte l'appel au vote communautaire en invitant les "Turcs" à voter pour "leurs propres candidats», ces injonctions ne se retrouvent pas dans les résultats du vote, particulièrement lors des élections locales. Entre autres raisons, la prépondérance de l'économie liée au tabac et les rapports de force qui en découlent. Une majorité de musulmans vit encore de la culture du tabac, dans les départements du Rhodope et de Xanthi, mais les coopératives agricoles sont dirigées par des Grecs ${ }^{96}$.

L'arène " turque » est l'objet de luttes de position qui en font un espace en constante redéfinition. L'apparent unanimisme lié au discours de la "turcité» masque des divergences, des affiliations partisanes concurrentes, comme en témoigne la victoire d' İlhan Ahmet aux élections législatives de 2004, alors qu'il n'était soutenu ni par le Conseil consultatif ni par le consulat de Turquie, mais individuellement par certains acteurs associatifs locaux et officiellement par le parti au pouvoir en Turquie. Dans le cadre des élections législatives, tout se passe en outre comme s'il y avait pour les électeurs musulmans un dédoublement de l'enjeu politique: ceux-ci opèrent un arbitrage en fonction de l'injonction au vote communautaire d'une part, selon les lignes de fracture entre partis nationaux, c'est-à-dire selon leur sensibilité partisane, de l'autre. L'intégration des musulmans au système politique grec se manifeste 
notamment par le fait que les clivages politiques qui lui sont propres semblent s'être peu à peu imposés aussi à eux. Il existe ainsi au sein de la population musulmane un système partisan qui suit une gradation droite/gauche, comme dans le reste de la Grèce, tout en se focalisant autant sur les partis que sur les personnalités, et sur leur positionnement par rapport à la question de la «minorité turque ». De ce fait, il ne faut pas négliger l'identification de nombres de ces acteurs aux partis politiques grecs: plusieurs de nos interlocuteurs militants de la cause «turque " montrent un profond attachement à un parti, en l'occurrence le PASOK (ou pour certains la gauche grecque, incarnée par le PASOK, le Synaspismos et le KKE). Ces personnes affirment que, quel que soit le candidat «turc » qui la représente, pour rien au monde ils ne donneraient leur voix à la Nouvelle Démocratie.

Il ne faut pas non plus négliger la variation des arbitrages effectués par les électeurs musulmans - fussent-ils militants de la cause «turque » - selon que le scrutin est de portée locale ou nationale. Ainsi, la secrétaire générale de l'Association des diplômés, qui fait partie des personnes dont on attend qu'elles soutiennent tel ou tel candidat " turc », a voté pour un candidat " grec » lors des élections locales de 2002. En effet, elle vote dans le village d'origine de son père, village mixte rattaché à une municipalité administrée par une équipe " grecque » apparentée communiste, ce qui correspond à la sensibilité politique de cette interlocutrice. Elle ajoute n'avoir jamais souhaité se faire enregistrer à l'état civil de sa commune de résidence, entièrement musulmane, car elle préfère s'adresser à des "Grecs » lorsqu'elle a des démarches administratives à faire, qui selon elle sont plus compétents que les "Turcs» en tant que gestionnaires municipaux. Notre interlocutrice a par ailleurs soutenu lors de ces élections la liste du candidat "grec» soutenue par le KKE à la mairie de Komotini, chef-lieu du département : un homme "fort sympathique » qui est venu rencontrer sa famille, et " très attentif aux questions concernant la minorité». Pour ce qui est des élections législatives, en revanche, la jeune femme trouve "l'enjeu bien trop important pour la minorité » et réprouve le fait que certains "Turcs » fassent défection à la cause de la minorité en votant pour des candidats "grecs $»^{97}$. On aurait tort, en somme, de se représenter les acteurs impliqués dans l'arène «turque » et les électeurs musulmans en général comme étant déterminés par la vie politique turque ; les enjeux qui occupent les acteurs du mouvement identitaire se situent dans un cadre politique grec, et avant tout local, quand bien même ce cadre local est marqué par la proximité et la présence historique de la Turquie dans la région.

\section{Annexe 1 : Liste des candidats musulmans/ »turcs » aux élections législatives du 7 mars 2004. Classement par parti et par position sur la liste}

\section{Rhodope}

NEA DIMOKRATIA (Nouvelle Démocratie) : İlhan Ahmet (1/4); Nazif Şakir (3/4)

PANHELLINIO SOSIALISTIKO KINIMA (PASOK, Mouvement socialiste panhellénique) : Galip Galip (1/4); Mehmet Ahmet (3/4)

SYNASPISMOS (Union des gauches) : Mustafa Mustafa (1/4) ; Ferhat Nazif (3/4)

KOMMOUNISTIKO KOMMA ELLADAS (KKE, Parti communiste de Grèce) : Nazim Ahmet $(1 / 4)$ 
DIMOKRATIO KINONIKO KINIMA (DIKKI - Mouvement démocratique et social) : Sadık İ zet $(1 / 4)$

ANTIKAPITALISTIKI SYMMAHIA (Alliance anticapitaliste) : Alim Hacı Hüseyin (1/1)

KOMMOUNISTIKO KOMMA ELLADAS (marxistiko-leninistiko) - (KKE-ML, Parti communiste de Grèce marxiste-léniniste) : Keke İsmet (1/3)

\section{Xanthi}

51 NEA DIMOKRATIA (Nouvelle Démocratie) : Türkeş Hacımemiş (4/4)

PANHELLINIO SOSIALISTIKO KINIMA (PASOK, Mouvement socialiste panhellénique) : Baran Burhan (1/4)

SYNASPISMOS (Union des gauches) : Ferhat Ramazan (4/4)

DIMOKRATIO KINONIKO KINIMA (DIKKI - Mouvement démocratique et social): Sebahaddin Toşunoğlu (2/3)

KOMMOUNISTIKO KOMMA ELLADAS (KKE, Parti communiste de Grèce) : İhsan Ahmet Kahya (1/4)

KOMMOUNISTIKO KOMMA ELLADAS (marxistiko-leninistiko) - (KKE-ML, Parti communiste de Grèce marxiste-léniniste): Muzaffer Kosoğlu (1/3); Mehmet Çakır $(3 / 3)$

\section{Annexe 2 : Le scrutin de liste pour les élections législatives en Grèce}

Le mode de scrutin appliqué lors des élections du 7 mars 2004 est issu de la Loi électorale du $1^{\mathrm{er}}$ janvier 1993. Sa particularité par rapport aux lois électorales précédentes est d'imposer aux partis l'obtention d'un minimum de $3 \%$ des suffrages pour leur entrée au Parlement. Les partis qui ont présenté des candidats dans $3 / 4$ au moins des circonscriptions du pays et obtenu un minimum de $3 \%$ des suffrages ont au minimum un siège assuré. Cette loi prévoit un scrutin de liste à un tour (représentation proportionnelle « renforcée ») avec répartition des sièges selon la méthode HagenbachBischoff. Les électeurs votent pour la liste d'un parti et ont la possibilité d'exprimer, en outre, leur préférence pour un candidat en apposant une croix devant son nom, indépendamment $\mathrm{du}$ rang de ce candidat sur la liste. Une première répartition des sièges a lieu qui est fonction de la "mesure électorale» c'est-à-dire le nombre minimum de voix que doit obtenir un candidat pour être élu dans son département (cette mesure est obtenue en divisant le nombre de voix obtenues dans chaque département par le nombre de sièges attribués au département plus 1).

Concrètement, voici comment la situation se présentait dans le Rhodope, qui avait droit à trois sièges. Pour obtenir un siège, il fallait qu'un parti totalise un quart des suffrages du département, puis était élu le candidat de ce parti qui avait réalisé le score le plus élevé de la liste. Si un parti totalisait plus de la moitié des voix, il avait deux élus. Dans le cas contraire, le deuxième parti ayant franchi le barrage électoral, envoie lui aussi son candidat ayant totalisé le plus de suffrages à l'assemblée. Dans le cas où aucun autre parti ne franchit le barrage, c'est le parti arrivé en tête qui obtient un deuxième siège pour son candidat ayant le deuxième meilleur score. Le troisième siège a été attribué lors de la seconde répartition des voix. Les sièges restants après la première répartition au niveau départemental sont redistribués au niveau régional (13 
circonscriptions régionales), selon le même système. Tout siège restant à l'issue de cette deuxième répartition est alors attribué au niveau national (une circonscription nationale plurinominale constituée par 12 « députés d'Etat»). Les 12 "députés d'Etat » sont proposés par les partis politiques les plus importants et élus au scrutin de liste avec répartition proportionnelle des sièges. En cas de vacance de siège en cours de législature, il est généralement fait appel au candidat de la même liste ayant réalisé le score immédiatement inférieur.

\section{Annexe 3 : Biographie des principaux candidats " turcs " et " grecs »}

Galip Galip (PASOK) : Le député sortant du Rhodope est issu d'une des plus grandes familles locales. Son grand-père avait été l'un des membres fondateurs de l'éphémère République de Thrace occidentale en 1913, son père fut député du Rhodope et parraina l'entrée en politique de Sadık Ahmet. Plusieurs membres de sa famille, dont son oncle paternel, furent également impliqués dans la gestion des biens de la communauté musulmane [cemaat]. Né en 1947 à Komotini, il a fait toute sa scolarité, primaire et secondaire, dans les établissements de la minorité. Il a suivi une formation d'architecte à l'Université technique d'Istanbul, dont il a obtenu le diplôme en 1972. En raison des problèmes de reconnaissance des diplômes que causent les autorités grecques aux membres de la minorité, ce n'est qu'en 1992 qu'il a commencé à exercer sa profession, jusqu'en 1996 où il a été élu député (et de nouveau depuis 2004). Entre-temps, il a travaillé à Komotini en tant que topographe, décorateur, et aussi dans les secteurs du tourisme et des assurances. Galip Galip n'avait pas pour ambition d'être député, mais étant donné ses antécédents familiaux, «tout le monde » a souhaité qu'il se présente aux élections après la disparition de Sadık Ahmet ${ }^{98}$.

İlhan Ahmet (ND) : Né en 1968, il a suivi les enseignements du lycée Celal Bayar durant la première partie du cycle secondaire (l'équivalent du collège ou ortaokul), puis il a terminé sa scolarité secondaire dans un lycée grec de Komotini. Il a obtenu le diplôme de la faculté de droit de l'Université d'Istanbul en 1993, et a commencé à exercer comme avocat en 1999. İlhan Ahmet a été élu membre de l'assemblée départementale $\mathrm{du}$ Rhodope sur la liste soutenue par la Nouvelle Démocratie en octobre 2002, mais seulement avec 3242 voix, le score le plus faible des huit conseillers élus dans l'opposition (la liste soutenue par le PASOK est arrivée en tête). Il est également depuis 2003 président du conseil de l'école primaire de la minorité du quartier dans lequel il a grandi et effectué sa scolarité (İdadiye Türk İlkokulu Encümen Heyeti Başkani).

Burhan Baran (PASOK) est également un très bon exemple de ce type de reconversion d'un capital social et universitaire en politique. Né en 1959 dans un village du département de Xanthi, il a effectué sa scolarité secondaire au lycée de la minorité de Xanthi (Lycée Muzaffer Salihoğlu) avant d'être admis à la faculté de médecine de l'Université d'Istanbul (où il a fait une spécialisation en oncologie). Il a enseigné quelque temps dans cette université, puis a fait un troisième cycle universitaire en Allemagne; il est rentré en Grèce en 1998 et a fait une deuxième spécialisation en psychiatrie. Il a été élu en 2002 à l'assemblée départementale de Xanthi avec 3800 voix, sur la liste soutenue par le PASOK. 
57 Euripidis Stilianidis(ND), député du Rhodope depuis 2000, réélu en 2004 et nommé peu après vice-ministre des Affaires étrangères, est titulaire d'un doctorat de droit constitutionnel obtenu à l'Université de Hambourg. Au cours de ses études de droit à l'Université Démocrite de Thrace, il a été de 1984 à 1989 le porte-parole de l'Union des étudiants au sein de la Nouvelle Démocratie. Il était parallèlement à cela membre du comité exécutif de l'Union nationale des étudiants. De 1991 à 1994, il a travaillé au Consulat général de Grèce de Hambourg. De 1994 à 1995, il a été le porte-parole du bureau exécutif de l'organisation de jeunesse de la Nouvelle Démocratie, avant de devenir le conseiller du président de la Nouvelle Démocratie sur les questions de la jeunesse et de la diplomatie culturelle. Elu au sein des instances de direction de la Nouvelle Démocratie en 1997, il a été parallèlement, jusqu'en 2000, enseignant chercheur au sein du département de droit public à l'Université d'Athènes. Il a été responsable de la section du parti pour les Affaires étrangères, particulièrement pour la région des Balkans, et s'occupait à ce titre des «questions nationales et juridiques relevant de la Défense nationale ${ }^{99}$.

Chrissa Manolia(PASOK) est diplômée du Département de littérature grecque de l'Université Aristote à Thessalonique ; elle a aussi étudié la littérature allemande. Elle a débuté sa carrière d'enseignante dans un lycée de Komotini en 1973. Elle a été secrétaire générale de l'Union départementale des enseignants du secondaire du Rhodope de 1983 à 1986. Elle a été parallèlement présidente de la Commission pour l'égalité entre les sexes de la préfecture du Rhodope (1984-1989), puis présidente de la Commission pour les femmes de la municipalité de Komotini (1993-1998), et à partir de 1999 présidente de la Commission régionale de Macédoine orientale-Thrace occidentale pour l'égalité entre les sexes. De 1991 à 2000 elle a eu un mandat de conseillère municipale, mais c'est en 1996 qu'elle a pris sa carte du PASOK. De 1996 à 1997, elle a été nommée secrétaire générale du ministère de la Macédoine et de la Thrace et en 2000 elle a été la première femme à être élue députée en Thrace ${ }^{100}$. Elle a perdu son siège quelques mois après suite à un recours intenté contre elle par son colistier Ahmet Mehmet pour cumul de mandats, car elle n'avait pas démissionné de son poste de conseillère municipale.

\section{Annexe 4 : Méthodologie et tableaux}

Pour le département de Xanthi, notre échantillon était constitué des données électorales concernant les municipalités de Mikis (Mustafçova), ainsi que des villages de Thèrmes (Ilica), Kotylis (Kozluca), Satrès (Sinikova). La municipalité de Mikis est la seule de toute la Thrace qui soit entièrement composée de villages exclusivement " musulmans ». Avec les autres villages de notre échantillon, elle forme un ensemble composé à $97 \%$ de « musulmans ${ }^{101}$. Pour le département du Rhodope, nous avons isolé les villages d'Arriana (Kozlukebir), Philira (Sirkeli), rattachés respectivement à deux municipalités mixtes éponymes, plus les villages d'Amaxades (Arabacıköy), Kehros (Mehrikoz) et Organi (Hemetli) ${ }^{102}$. Nous avons été obligées de faire coexister deux échelons municipaux, celui de municipalité [demos] et de commune [koinotita] afin d'obtenir des échantillons suffisamment importants numériquement pour être représentatifs. Il est en revanche impossible d'isoler les votes «musulmans » dans les centres urbains de Komotini et Xanthi, dans la mesure où, depuis 1996, les listes électorales et les bureaux de vote ne séparent plus les « musulmans » des " chrétiens ». 
Il y a bien des bureaux de vote dont on sait qu'ils sont fréquentés en majorité par des " musulmans", mais nous n'avons pas pu obtenir les chiffres de chaque bureau de vote sur l'ensemble des trois élections législatives, à la fois pour Xanthi et Komotini.

De la même façon, nous connaissons les taux d'abstention à l'échelle départementale (voir les tableaux joints) pour l'ensemble de l'électorat, mais nous ne pouvons la mesurer pour l'électorat "musulman». Concernant nos échantillons, nous avons travaillé directement sur le nombre de votants, sans fournir un pourcentage du taux d'abstention. Nous l'avons dit, nos objectifs en termes d'analyse quantitative sont somme toute modestes, notre attention s'est portée en priorité sur la concordance entre le vote "turc » et l'électorat "musulman ». On voit à l'échelle départementale que le taux d'abstention est stable entre les trois scrutins à Xanthi, et que dans le Rhodope il baisse de près de quatre points entre 1996 et 2004. Néanmoins, considérant qu'il est toujours compris entre 27 et $30 \%$, et considérant la forte mobilisation des électeurs «musulmans » non résidants ${ }^{103}$, nous avons pris le taux d'abstention comme un seuil incompressible et comme une donnée secondaire pour laquelle nous ne disposions ni d'hypothèse ni d'éléments d'analyse pertinents. Précisons que cette importante mobilisation n'est pas seulement le fait des électeurs "musulmans " puisque le nombre de vote exprimés à Xanthi et dans le Rhodope, à l'échelle départementale, a considérablement augmenté : environ 10000 votes supplémentaires ont été exprimés à Xanthi en 2004 par rapport à 1996 (pour seulement 1400 électeurs de plus en 2000); dans le Rhodope, l'augmentation du nombre de votants est d'environ 7 000 en 2004 par rapport à 1996 (le nombre de votants avait baissé en 2000). Le taux d'abstention n'a baissé de façon significative que dans le Rhodope au cours de cette même période.

61 Rappelons que les commentateurs locaux ont tendance à confondre la population «musulmane» (donnée elle-même inconnue en raison de l'absence de statistiques officielles depuis le recensement de 1951), et le nombre d'électeurs "musulmans». Considérons que selon les scrutins et les sources sollicitées, le nombre d'électeurs musulmans du département de Xanthi est compris dans une fourchette de 25 à 35000 , et celui des électeurs musulmans du département du Rhodope dans une fourchette de 35 à 45 000. En 2004, d'après le journal turc Vatan, les candidats grecs tablaient sur environ 70000 votes «musulmans $»^{104}$. D'après Ilhan Ahmet, qui nous a fourni cette donnée peu après son élection, il y a eu 42800 votes «musulmans » exprimés lors des élections législatives de 2004. Cela nous permet en outre de supposer que les partis politiques et candidats locaux connaissent leur électorat et la structure de celui-ci, et que des statistiques sont réalisées mais non diffusées; Chrissa Manolia par exemple peut évaluer son électorat "musulman". Considérant la mobilisation massive et inédite des électeurs venus de Turquie et d'Allemagne, ayant abouti à une augmentation des votes «musulmans» en valeur absolue, nous avons retenu pour les scrutins précédents, afin de pouvoir procéder à une comparaison, l'estimation de 35 000 électeurs «musulmans » dans le Rhodope, et 25000 à Xanthi ${ }^{105}$. Ce flou des données est gênant pour une analyse statistique précise, que nous n'avons pas eu les moyens de mettre en œuvre ici, mais étant donnés nos objectifs plus modestes, il nous donne une indication de la taille de l'électorat "musulman ». Bien sûr, les chiffres pris en compte ne sont pas de même nature: à l'échelle du département, il ne s'agit que de l'estimation mentionnée plus haut. En revanche, le pourcentage établi à partir de nos échantillons a été calculé à partir de données exactes. 
4.1. Tableaux par département (voir liens proposés en « Documents annexes » au format PDF)

\section{BIBLIOGRAPHIE}

Aarbakke, Vemund (2000) The Muslim Minority of Greek Thrace, Thèse de doctorat, Bergen.

Akgönül, Samim (1999) Une communauté, deux Etats : la minorité turco-musulmane de Thrace occidentale, Istanbul, Isis

Anastassiadou, Méropi (1992) « Compte-rendu de l'ouvrage de Symeon Soltarides : La Thrace occidentale et les musulmans, que se passe-t-il exactement? ", Lettre d'information du groupe de travail sur la transmission du savoir dans le monde musulman périphérique, 12, pp. 76-79.

Becker, Howard (1985) [1963] Outsiders. Études de sociologie de la déviance, Paris, Editions Métailié. Bonos, Yannis (2007) « The episode of the Turkish spelling mistakes in Greek Thrace, June 1929: beyond the opposition of modernists and conservatives » in Clayer, Nathalie; Germain, Éric (eds) Islam in Europe in the inter-war period. Networks, status, challenges (à paraître), pp. 362-386.

Bourdieu, Pierre (1980) Le sens pratique, Paris, Editions de Minuit.

Centlivres, Pierre; Fabre, Daniel; Zonabend, Françoise (dir.) (1998) La fabrique des héros, Paris, Editions de la Maison des sciences de l'homme.

Copeaux, Étienne (1999) «Linguistique et discours historique : premier bilan et pistes de recherche ", Les mots du politique de l'Empire ottoman à la Turquie kémaliste, Études turques et ottomanes, documents de travail, $\mathrm{n}^{\circ} 8, \mathrm{pp} .7-19$. 
Diamantopoulos, Thanassis (2003) « La Grèce post-dictatoriale : forces politiques et opinion publique », Pôle Sud, La Grèce du politique, 18, pp. 31-50.

Dezalay, Yves; Garth, Bryant (2002) La mondialisation des guerres de palais. La restructuration du pouvoir d'État en Alérique latine, entre notables du droit et "Chicago Boys », Paris, coll. Liber, Seuil.

Fretel, Julien (2004) « Le parti comme fabrique de notables. Réflexion sur les pratiques notabiliaires des élus de l'UDF », Politix. Trajectoires de la notabilité, I. Pratiques et stratégies, 65, pp. 45-72.

Gaïtti, Brigitte (1998) De Gaulle, prophète de la Ve République, Paris, Presses de Sciences Po.

Gaxie, Daniel (1978) Le cens caché, Paris, Le Seuil.

Gaxie, Daniel (dir.) (1989) Explication du vote. Un bilan des études électorales en France, Paris, Presses

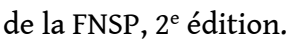

Hersant, Jeanne (2000) «L'évolution de la question de la minorité musulmane de Thrace occidentale dans le cadre de l'intégration européenne de la Grèce », mémoire de fin d'études sous la direction de Gilles Dorronsoro, IEP de Rennes.

Hersant, Jeanne (2005a) «Transmission de l'identité et culte du héros : les associations de Turcs de Thrace occidentale en Allemagne », Balkanologie, IX (1-2), pp. 103-130.

Hersant, Jeanne (2005b) « Les associations de migrants originaires de Thrace occidentale (Grèce) à Bursa et Zeytinburnu : pratiques politiques et figures du notable » in Hersant, Jeanne; Toumarkine, Alexandre (eds) Hometown Organisations in Turkey, European Journal of Turkish Studies, Thematic Issue $\mathrm{n}^{\circ} 2$, http://www.ejts.org/document368.html

Hersant, Jeanne (2007) « Mobilisations politiques, co-gouvernementalité et construction ethnique. Sociologie du nationalisme turc à travers le cas de Turcs de Thrace occidentale (Grèce, Allemagne, Turquie) ", thèse de sociologie politique sous dir. Hamit Bozarslan, EHESS.

Hlepas, Nicolas-Komnenos (2003) « Carte administrative et pouvoir politique : les enjeux de la décentralisation en Grèce », Pôle Sud, La Grèce du politique, 18, pp. 63-78.

Labrianidis, Lois (1999) “The Impact of the Greek Military Surveillance Zone on the Greek Side of the Bulgarian-Greek Borderlands", Boundary and Security Bulletin, 7 (2), pp. 82-93.

Lagroye, Jacques ; François, Bastien; Sawicki, Frédéric (2002) Sociologie politique, Paris, Presses de Sciences Po/Dalloz, $4^{\mathrm{e}}$ édition.

Lahire, Bernard (dir.) (2001) Le travail sociologique de Pierre Bourdieu. Dettes et critiques, Paris, La Découverte, $2^{\mathrm{e}}$ édition.

Lamprini, Rori (2007) «Les procédures délibératives et le clientélisme de parti : l'exemple du PASOK », communication présentée lors de la journée d'études « Les partis politiques à l'épreuve des procédures délibératives », IEP de Toulouse (Lassp), 15 juin 2007.

Marantzidis, Nikos; Mavromatis, Georges (2000) «Identité ethnique et comportement politique : le comportement électoral d'un groupe de tsiganes musulmans en Thrace occidentale », Etudes helléniques 8 (1), pp. 71-97.

Massicard, Elise (2003) «Les élections du 3 novembre 2002 : une recomposition de la vie politique turque ? », Les dossiers de l'IFEA, $\mathrm{n}^{\circ} 15$.

Massicard, Elise (2004) «Entre l'intermédiaire et l'homme d'honneur. Savoir-faire et dilemmes notabiliaires en Turquie », Politix. Trajectoires de la notabilité II, 67, pp. 101-127.

Massicard, Elise (2005) L'autre Turquie, Paris, PUF. 
Mavrogordatos, Giorgos (1983) Stillborn Republic. Social Coalitions and Party Strategies in Greece (1922-1936), California, University of California Press.

Mayeur-Jaouen, Catherine (dir.) (2002) Saints et héros du Moyen-Orient contemporain, Paris, Maisonneuve \& Larose.

Nikolakopoulos, Ilias (1990-91) « Forces politiques et comportement électoral de la minorité musulmane de Thrace occidentale 1923-1955 » (en grec), Bulletin du Centre d'Etudes sur l'Asie Mineure, t. IX.

Sotiropoulos, Dimitri A.; Borikas, Dimitri (2003) « Les élites ministérielles en Grèce, de 1974 à l'an 2000 », Pôle Sud, La Grèce du politique, 18, pp. 79-89.

Tudesq, André-Jean (1989) « Les comportements électoraux sous le régime censitaire » in Daniel Gaxie (dir.) (1989) Explication du vote. Un bilan des études électorales en France, Paris, Presses de la FNSP, $2^{\mathrm{e}}$ édition, pp. 106-125.

Zulfikarpasic, Adélaïde (2001) « Le vote blanc : abstention civique ou expression politique ?», Revue française de science politique, 51 (1-2).

\section{NOTES}

1. Ce traité leur a en outre procuré des droits positifs par rapport aux autres citoyens grecs, notamment la possibilité d'être soumis à la loi islamique en matière de juridiction civile : c'est le mufti, chef religieux, qui statue sur les questions de mariage, divorce ou héritage, ses actes étant simplement contresignés par l'administration grecque.

2. Pour ce qui est des instituteurs et du personnel religieux, plus les retraités, il s'agit d'environ 1500 personnes recevant un salaire d'environ 1000 euros par mois pour les postes les plus élevés (Entretien réalisé avec un ancien journaliste de Gündem, janvier 2002). Ce chiffre ne prend pas en compte le personnel associatif et les subventions à la presse locale, sommes difficiles à évaluer car ce financement n'est jamais admis publiquement.

3. Sur la définition d'un mouvement identitaire, voir Massicard (2005).

4. Ce contentieux porte notamment sur Chypre et sur la délimitation des eaux territoriales en mer Égée (Bertrand 2004).

5. Le troisième département, celui de l'Evros, a perdu la quasi-totalité de sa population musulmane avant même l'échange de population ; celle-ci n'y est pas suffisamment importante pour avoir un poids électoral ou élire des représentants, même au niveau local. Le nombre de députés par nomos a été décidé en fonction du recensement de 2000. Le département de l'Evros, le plus peuplé, a droit à quatre députés, tandis que ceux du Rhodope et de Xanthi en ont chacun trois. Source : Rıza Kırlıdökme, «Kaynayan Seçim Kazanında " [Dans le chaudron en ébullition des élections], Gündem, 13/02/04.

6. Cette population n'est pas exclusivement turcophone, elle compte également de nombreux locuteurs du pomaque, dialecte du bulgare; néanmoins le turc est la langue véhiculaire au sein de la « minorité musulmane ».

7. Les élections du 7 mars 2004 ont eu lieu un an avant la date initialement prévue. À l'approche du référendum sur le plan Annan à Chypre et des jeux Olympiques d'Athènes, le Premier ministre Kostas Simitis (PASOK) a voulu conforter sa majorité parlementaire. Hülya Emin, «Seçimlere dolu dizgin... » [Vers les élections à bride abattue], Gündem, 09/01/04

8. Dans l'après-guerre et jusqu'aux années 1960 , il y eut en revanche jusqu'à quatre députés issus de cette minorité, trois le plus souvent (deux dans le Rhodope et un à Xanthi). 
9. Les chiffres avancés pour quantifier la «minorité musulmane» sont également des estimations : de 120000 pour les autorités grecques à 150000 pour les membres du mouvement identitaire et les autorités turques.

10. Le bureau du SYNASPISMOS à Xanthi nous a gracieusement envoyé des copies de l'ensemble de ses archives concernant les élections sur la période récente.

11. Les termes associés à la pathologie sont fréquemment employés pour décrire les rapports entre membres de la minorité et société environnante, mais plus particulièrement au sujet des Pomaques qui seraient « déchirés » entre plusieurs identités, victimes à la fois des Grecs et des Turcs. Ibrahim Onsunoğlu, psychiatre de Thessalonique issu de la "minorité », ancien militant d'extrême gauche aux ambitions politiques déçues en Thrace, affirme que la dépression (qu'il met en parallèle avec la "paranoïa grecque ») est un phénomène généralisé au sein de la minorité, particulièrement chez les femmes. Didier Kuntz et Daniel Vernet, « Musulman, c'est possible, mais dur », Le Monde, 08/08/04. À notre connaissance aucune étude systématique n'a été menée sur le sujet.

12. Cette libéralisation s'est manifestée, entre autres choses, par le démantèlement total, au début des années 2000, de la zone militaire fermée qui enserrait une grande partie du territoire de la Thrace occidentale. Pour ce qui est du mouvement de protestation antérieur au mouvement identitaire "turc", qui visait à dénoncer les discriminations subies par les musulmans, en Allemagne et en Grèce, voir Hersant (2007). Aujourd'hui, ce discours est largement rhétorique, néanmoins les autorités grecques continuent d'interdire les associations de la minorité dont le nom fait référence à la turcité.

13. Sur la fabrique des héros, saints et martyrs, voir Pierre Centlivres et alii (1998); Catherine Mayeur-Jaouen (2002). Pour plus de détails sur le culte du héros rendu à Sadık Ahmet, voir Hersant (2005).

14. La notion de champ du pouvoir d'État est définie par Dezalay et Garth (2002).

15. Comme l'illustre cet incident, lors d'une soirée de gala organisée par l'Association des membres de la minorité diplômés des universités [Batı Trakya Azınlı̆ı Yüksek Tahsilliler Derneği], dans le cadre de la campagne pour les élections locales : le maire sortant de Komotini et candidat à sa succession n'a pas pu prendre la parole et a quitté la salle en signe de protestation, alors que le consul général de Turquie avait été invité à faire un discours. "BTAYTD'den yardımlaşma gecesi » [Le gala de solidarité de l'Association des diplômés (ici désignée par son acronyme)], Gündem, 24/09/02; pour plus de précisions, voir (Hersant 2007).

16. Pour une définition du champ, voir par exemple Pierre Bourdieu (1980), Bernard Lahire (2001), Jacques Lagroye etalii (2002).

17. Ce terme se réfère à des organisations (partis, mouvements, etc.) qui se caractérisent par leur participation directe à la compétition pour des postes et des positions de pouvoir politique. Jacques Lagroye etalii (2002: 225).

18. Depuis le milieu des années 1980 coexistent en Thrace deux muftis nommés par Athènes, qui sont fonctionnaires grecs, et deux muftis dissidents élus au sein de la minorité, qui eux sont rémunérés par le gouvernement turc. Pour plus de précisions sur ce point, voir Bonos (2007) et Hersant (2007).

19. L'Association des instituteurs turcs de Thrace occidentale, l'Union turque de Xanthi, l'Union des jeunes Turcs de Komotini.

20. Expression consacrée faisant référence aux litiges opposants les représentants de la minorité aux autorités grecques, notamment l'interdiction dont sont l'objet les associations portant l'adjectif «turc» dans leur intitulé, ou encore la question de la gestion des vakıf (ou biens de mainmorte) liée à celle de l'élection des mufti.

21. Prenons l'exemple de Çetin Mandacı, qui était en 2004 président de l'Union turque de Xanthi et détenteur d'un mandat local; il a été élu député de Xanthi lors des élections législatives de 
septembre 2007. Son successeur à la tête de l'Union turque de Xanthi est également journaliste à Gündem, de même que le président de l'Association des diplômés de la minorité.

22. On trouvera les détails de la création et de la polémique qui ont entouré ce parti et son fondateur dans Aarbakke (2000) et Akgönül (1999). Ahmet Haciosman a été élu en 2002 membre de l'assemblée départementale du Rhodope au sein de la liste commune PASOK-Synaspismos. Avec 13701 voix, il est le candidat qui a obtenu le plus grand nombre de voix de tous les candidats («grecs » et "turcs » confondus) des deux départements du Rhodope et de l'Evros réunis, qui constituent une sous-région administrative. Ahmet Haciosman a ainsi obtenu plus du double de voix du candidat " grec » ayant obtenu le meilleur score sur la même liste, en troisième position avec 6004 voix. Il est député du Rhodope depuis les élections législatives anticipées de septembre 2007.

23. PASOK, Mouvement socialiste panhellénique; KKE, Parti communiste grec; Synaspismos, Coalition de la gauche et du progrès, issu d'une scission au sein du KKE dans les années 1990. À la veille des élections de 2007, cette formation est devenue Syriza (Coalition de la gauche radicale).

24. Il s'agit notamment des différentes déclinaisons des partis marxistes-léninistes, qui ont réalisé en 2004 des scores quasiment nuls. Ceux-ci ont quand même été comptabilisés et publiés dans Gündem, en tant que votes pour les candidats " turcs ». " Rodop ili 2004 seçim sonuçları », Gündem, 19/03/2004.

25. Pour plus de précisions sur le maillage institutionnel par lequel la Thrace occidentale est maintenue sous tutelle politique et militaire, voir Hersant (2007).

26. Entretien réalisé à Komotini, juillet 2003.

27. Par exemple, İlhan Ahmet nous a affirmé qu'un député du Parti de la justice et du développement (AKP), la nouvelle majorité gouvernementale en Turquie était venu à Komotini le soutenir quelques jours avant le scrutin ; il n'en a pas été question dans Gündem.

28. A la veille des élections, deux cadres historiques de la Nouvelle Démocratie, Manos et Andhrianopoulos, ont rejoint les rangs du PASOK, alors que Papathemelis, personnalité de l'aile nationaliste du PASOK, a intégré la ND. Maria Damanaki, qui est un des cadres fondateurs du Synaspismos et qui en a été secrétaire générale durant de nombreuses années, a elle aussi rejoint le PASOK.

29. «Hacımemiş, YDP'den milletvekili aday adayı " [Hacımemiş candidat à l'investiture au sein de la ND], Gündem, 16/01/04. En Grèce, c'est une somme confortable par rapport au revenu moyen. A titre d'exemple, en 2003 le salaire minimum était de 473 euros en Grèce et de 1126 euros en France. Source : Gérard Filoche, "L'Europe et le SMIC unique européen », 4 juin 2004, http://www.democratie-socialisme.org/article.php3?id_article $=415$.

30. Il est le premier musulman devenu psychiatre, et ne fait plus partie aujourd'hui du personnel politique local. D'abord militant d'extrême gauche, il est le seul musulman et la seule personne de Komotini à avoir participé à l'insurrection de l'École polytechnique d'Athènes, le 17 novembre 1973, lorsqu'il était étudiant. Il fut ensuite parmi les cadres fondateurs du PASOK dans le département du Rhodope, puis il quitta ce parti à la fin des années 1980, sans nous en donner explicitement la raison. Il fit un bref retour en politique au début des années 1990, principalement en opposition à Sadık Ahmet, au sein de la Nouvelle Démocratie, mais il essuya un échec et abandonna la politique.

31. Entretien réalisé en décembre 2004.

32. Nous remercions Gerassimos Moschonas d'avoir précisé ce point.

33. «Seçim maratonu başladı » [Le marathon électoral a commencé], Gündem, 13/02/04.

34. Entretien réalisé en décembre 2004.

35. Sur le clientélisme au sein du PASOK, voir Rori Lamprini (2007).

36. 'Seçim propagandası - Televizyon ve azınlığın talepleri' [Campagne électorale - Télévision et demandes de la minorité], Gündem, 23/01/04. 
37. "Seçimde en kritik rolü Batı Trakya oynayacak» [Le rôle de la Thrace occidentale sera déterminant dans les élections], Vatan, 07/03/04 ; Gündüz Aktan, «Böyle giderse, Batı Trakya » [Si la Thrace occidentale continue comme ça....], Radikal, 01/03/04.

38. Parti de la justice et de développement, au pouvoir en Turquie depuis 2002.

39. Il est par ailleurs l'ancien président de l'Union turque de Xanthi, et le propriétaire et «proviseur » du lycée Muzaffer Salihoğlu de Xanthi.

40. Voir par exemple Hülya Emin «Türk vekillerle yola devam» [Nous poursuivons notre chemin avec les représentants turcs], Gündem, 27/02/2004; Cemil Kabza, «Kararsılılı devam ediyor » [L'indécision continue], Gündem, 27/02/2004. Concernant la rhétorique du nationalisme turc, voir les travaux d'Étienne Copeaux, notamment (Copeaux 1999).

41. Après la victoire électorale des candidats "turcs" sans étiquette, et suite à la période d'instabilité gouvernementale de 1989-1990, la loi électorale a été modifiée et prévoit désormais que pour être représenté au Parlement, un candidat ou un parti doit obtenir plus de $3 \%$ des voix à l'échelle nationale.

42. Sur ce point on se reportera à la conceptualisation par Max Weber du type charismatique de la domination légitime. On peut aussi effectuer une analogie avec la thèse de Brigitte Gaïti (1998) sur la notion de prophète, dans la mesure où toute l'histoire politique de la " minorité » est relue rétrospectivement à l'aune de la figure héroïque de Sadık Ahmet.

43. «Milletvekili adayları ne diyor?» [Que disent les candidats à la députation?], Gündem, 20/02/2004.

44. « Kaynayan seçim kazanında - 3 » [Dans le chaudron en ébullition des élections - 3], Gündem, 27/02/2004.

45. "Milletvekili adayları basın mesnuplarıyla biraraya geldi », [Réunion entre les candidats et les membres de la presse], Gündem, op.cit.

46. http://www.amb-grece.fr/grece/legislatives.htm

47. « Tout le monde sait que la minorité est très peu organisée politiquement, à travers des organisations. Les individus qui composent cette société n'ont pas été membres, ne sont pas entrés dans les partis politiques qui sont actifs dans le pays. (...) Or une société qui adopte un comportement de "parasite" ne pourra naturellement pas s'intégrer aux institutions». "Azınlık adaylarına aktif destek » [Soutien actif aux candidats de la minorité], Gündem, 20/02/2004.

48. «Yunan adaylar cami kapısında oy istedi » [Les candidats grecs prospectent à la porte des mosquées], Zaman, 07/03/2004.

49. Sur la notabilité, voir (Tudesq 1989; Fretel 2004), et sur le cas particulier de la Turquie (Massicard 2004).

50. Galip Galip a longuement répondu à ce grief dans son dernier meeting électoral, l'avantveille du scrutin, y répondant par son intense activité en faveur de la promotion des droits de la « minorité turque ». " Galip Galip : işe eğitim sorunlarımızdan başladık» [Galip Galip : nous nous sommes occupés en priorité de notre problème d'éducation], Gündem, 05/03/2004.

51. «Rodoplu'dan seçmen ve adaylara mesaj...»[Le message de Rodoplu aux électeurs et aux candidats], Gündem, 10/02/2004.

52. «Bir milletvekilinin güncesi » [L'emploi du temps d'un député], Gündem, 09/07/04.

53. Entretien à Komotini, août 2002. En réalité les musulmans qui ne parlent pas le grec sont les personnes âgées et les habitants des villages non mixtes éloignés de la ville. Les citadins sont souvent parfaitement bilingues.

54. La différence étant que les candidats « grecs » se rendent dans tous les villages, tandis que les candidats « turcs » s'adressent quasi exclusivement à l'électorat musulman.

55. «Batı Trakya'da heyecan » [Suspense en Thrace occidentale], Milliyet, 29/02/04

56. «Meriç'in öte yakası : Batı Trakya Türkleri » [De l'autre côté de l'Evros : les Turcs de Thrace occidentale], Atlas, décembre 2003, p.182.

57. Nous l'avons suivie dans ces visites à Philira (Sirkeli), en septembre 2003. 
58. Entretien réalisé en mars 2004.

59. «Batı Trakya'da heyecan », Milliyet, op.cit. Cela n'est pas sans rappeler les pratiques similaires observables en Turquie, par exemple à l'occasion de la campagne pour les élections municipales de mars 2004 ; « İzmir'deki seçimi Genç Parti oyları belirleyecek » [Ce sont les voix du Parti jeune qui vont déterminer l'issue du scrutin à İzmir], Zaman, 19/03/04. Il y est question de la campagne du maire CHP de la ville, Ahmet Piriștina (réélu mais décédé d'une crise cardiaque quelques semaines plus tard) qui distribuait bonbons, dentifrice et brosses à dents.

60. Entretien réalisé en mars 2004.

61. Rappelons que les langues grecque et turque ont deux alphabets différents, grec et latin.

62. Entretien avec une membre de l'Association des diplômés, mars 2004.

63. Sur le rôle du dîner dans les rituels sociaux liés aux stratégies notabiliaires, voir Hersant (2005b).

64. Littéralement "vendeur de soupe », qui s'appliquait sous l'Empire ottoman aux notables grecs qui avaient des responsabilités dans l'administration. Cette précision est fournie par Altan Gökalp (2004) dans sa traduction du roman de Yaşar Kemal, Regarde l'Euphrate charrier le sang.

65. «İlhan Ahmet: Bireysel tanımlama hakkı yetmez» [İlhan Ahmet: la reconnaissance individuelle (de l'identité turque, ndt) ne suffit pas], Gündem, 05/03/2004.

66. Entretien réalisé en décembre 2004.

67. «Batı Trakya Türk azınlığınıb büyük sınavı »[La grande leçon donnée par la minorité turque de Thrace occidentale], Gündem, 05/03/04.

68. Cette victoire du parti conservateur était à ce point prévue, anticipée et attendue par le gouvernement turc que, toujours d'après les informations fournies par İlhan Ahmet, le premier ministre Tayyip Erdoğan aurait appelé Konstantin Karamanlis pour le féliciter de sa victoire plusieurs heures avant la publication officielle des résultats.

69. Entretien réalisé en décembre 2004.

70. La loi grecque ne prévoit pas le vote par procuration ni le vote depuis les représentations diplomatiques à l'étranger. Ainsi, bien que le vote soit théoriquement obligatoire en Grèce (les peines ne sont pas appliquées dans les faits pour les abstentionnistes), les résidants à l'étranger ne sont pas concernés par cette obligation, ce qui rend d'autant plus spectaculaire la mobilisation observée. Le vote depuis les consulats grecs est néanmoins en discussion depuis fin 2006. Voir par exemple : «Congres du conseil de l'hellénisme de la diaspora : M. Karamanlis annonce le droit de vote des Grecs de l'étranger»: http://www.info-grece.com/modules.php? name $=$ News\&file $=$ article $\&$ sid $=3543$

71. Le maire de Komotini, Tasos Vavatziklis, candidat à sa propre succession sur une liste soutenue par le PASOK, s'est lui aussi rendu en Turquie quelques jours avant le premier tour des élections. La raison officielle en était le projet de jumelage entre Komotini et Yalova, dans le cadre d'un programme européen de «lutte commune contre les catastrophes naturelles». Puis, avec la délégation qui l'accompagnait, il a rencontré les Batı Trakyalı de Yalova, avant d'aller rendre visite au maire de l'arrondissement de Yıldırım, à Bursa, distante d'une soixantaine de kilomètres. « Kardeşlik ilerliyor » [La fraternité progresse], Gündem, 08/10/02.

72. "Yunan seçimlerine otobüsle oy taşındı" [Pour les élections grecques, les votes ont été acheminés en bus], Yeni şafak, 16/10/2002.

73. "Seçimde en kritik rolü Batı Trakya oynayacak» [La Thrace occidentale jouera un rôle déterminant dans les élections], Vatan, 07/03/2004; Gündüz Aktan, «Böyle giderse, Batı Trakya... » [Si la Thrace occidentale continue comme ça...], Radikal, 01/03/2004.

74. Batı Trakya Türkleri Dayanışma Derneği, BTTDD.

75. Entretien réalisé à Küçükçekmece (Istanbul), février 2004.

76. Bien qu'elle n'ait pas de fondement rationnel, le fait que cette rumeur soit considérée comme vraie reflète l'attitude des autorités consulaires grecques à l'égard des membres de cette minorité en Turquie : contrairement aux autres ressortissants grecs et aux membres de la même minorité 
en Allemagne, ils ont l'obligation de retourner en Grèce lorsqu'ils doivent faire renouveler leurs papiers d'identité et ne se voient délivrer un passeport que pour un an, et non pour cinq (Hersant 2007).

77. «Batı Trakya'ya 'oy' yolculuğu », Türkiye, op.cit.

78. Cette estimation est fondée sur les déclaration du propriétaire d'une agence de voyages de Bursa, originaire de Thrace occidentale, qui a fourni six bus à l'Association de solidarité des Turcs de Thrace occidentale, à destination de Komotini et Xanthi, pour un montant de 6,6 milliards de livres turques (entretien réalisé en mars 2004). Cette somme correspond depuis janvier 2005 à 6600 YTL (Yeni Türk Lirası, Nouvelle Livre turque), soit environ 4000 euros à l'époque.

79. «Batı Trakya'ya 'oy' yolculuğu » [Le voyage des « votes » vers la Thrace occidentale], Türkiye, 05/03/04; «Batı Trakya'ya otobüsle 8000 oy gitti » [8 000 votes ont pris le bus pour la Thrace occidentale], Zaman, 06/03/04. D'après l'un des acteurs associatifs de l'arrondissement de Zeytinburnu (Istanbul), la douane aurait enregistré 11000 passages (il peut s'agir des allers et retours). Le président de l'association de Bursa quant à lui avançait le chiffre de 6000 . Nous avons choisi le nombre de 8000 , car le plus cité dans la presse, et médian entre les affirmations de nos interlocuteurs.

80. Il est très difficile de quantifier le nombre de musulmans de Thrace occidentale vivant en Turquie, et plus particulièrement ceux qui ont conservé la nationalité grecque : nombre d'entre eux vivent en Turquie avec un visa touristique, conformément aux accords de libre circulation entre l'Union européenne et la Turquie, qui autorise les ressortissants européens à séjourner trois mois consécutifs en Turquie sans autre justificatif qu'une carte d'identité ou un passeport.

81. Avrupa Batı Trakya Türk Federasyonu.

82. En tout 450 avions ont été affrétés par ces deux partis pour rapatrier les électeurs résidant dans les pays voisins de la Grèce, notamment de Chypre d'où 10000 électeurs seraient venus. «Seçimde en kritik rolü Batı Trakya oynayacak», Vatan, op.cit.; "Atina'ya hanedan beğenecekler » [Ils vont choisir une dynastie pour Athènes], Radikal, 07/03/04.

83. Nos interlocuteurs en Allemagne disent effectuer un voyage par an ou tous les deux ans, en famille et en voiture, pour la saison estivale, jamais en avion.

84. Des entretiens menés à Istanbul durant la période préélectorale ressortait une constante : le devoir dont se sentaient investis nos interlocuteurs, non pas tant d'aller voter que de voter pour un « Turc » s'ils faisaient le déplacement.

85. «Yunan adaylar cami kapısında oy istedi » [Les candidats grecs ont sollicité les votes à la porte des mosquées], Zaman, op.cit.; «Seçim maratonu başliyor...» [Le marathon électoral a commencé], Gündem, op.cit.

86. En 1964 déjà, puis en 1974 et en 1981, il n’y avait pas eu de député de la minorité dans le département de Xanthi.

87. Il y avait, en 2004, 114993 électeurs inscrits dans le Rhodope, et 108632 à Xanthi. Pour connaître l'évolution de ces chiffres, et de la participation, depuis 1996, on se reportera aux tableaux en annexe.

88. Ces chiffres, comme ceux concernant les électeurs venus de l'étranger, ne sont pas publiés. Ils nous ont été communiqués de façon officieuse par İlhan Ahmet, élu député du Rhodope dans les rangs de la Nouvelle Démocratie. Nous les tenons pour fiables (en admettant qu'ils puissent avoir été arrondis) dans la mesure où il est évident que les candidats et élus « turcs » tiennent des statistiques concernant l'électorat turc, même si celles-ci restent à usage interne. De la même façon, comme nous le verrons, les candidats " grecs » savent précisément évaluer leur électorat «musulman ». Nous n'avons pas pu obtenir de données équivalentes pour le département de Xanthi, pour lequel nous devrons nous contenter d'estimations.

89. Cette municipalité est composée de trois circonscriptions. La première regroupe les villages d'Ehinos (şahin) et Manevia (Elmalı) qui constituaient avant la réforme Capodistrias la commune d'Ehinos, soit sept bureaux de vote. La seconde circonscription correspond à l'ancienne 
commune de Mikis, soit les villages de Mikis (Mustafçova) et Kendavros (Ketenlik), et comprend 17 bureaux de vote; la troisième circonscription correspond à l'ancienne commune d'Oreon (Yassiören) constituée d'un seul village éponyme, et comptant quatre bureaux de vote.

90. Notons cependant que le DIKKI ne totalise que 0,63\% des votes exprimés au sein de cet échantillon (voir tableau en annexe).

91. Se reporter aux annexes pour une présentation plus détaillée de ce mode de scrutin.

92. « 7 Mart milletvekili seçimlerini biraz daha açalım» [Regardons d'un peu plus près les élections législatives du 7 mars], Gündem. Il y a sous ce titre huit éditoriaux datés du 12 mars au 30 avril 2004.

93. En 2004, le nombre de voix accordé aux candidats "grecs» y dépasse d'une cinquantaine celui pour les candidats "turcs». En 1996, par comparaison, dans la même municipalité le nombre de votes pour les candidats «turcs " était plus de deux fois supérieur à celui pour les candidats « grecs»; en 2000, l'écart de voix entre les candidats « turcs » et « grecs » n'était plus que de 528.

94. Nombre de musulmans de Thrace partis en Turquie effectuer leur scolarité secondaire et leurs études supérieures, particulièrement dans les années 1970 et 1980, n'ont jamais pu revenir s'établir en Grèce faute d'en parler la langue (Hersant 2007).

95. Damon Damianos, « Pourquoi la ND a gagné dans le Rhodope », Paratiritis, 12/03/04 (en grec). 96. A titre d'exemple, le député de Xanthi, Alekos Kontos, est également le vice-président du conseil d'administration de la SEKE (Synetairistiko Ergostaio Epexergasias Kapnou, Usine coopérative de traitement du tabac).

97. Cette hiérarchie très nette opérée par les électeurs ayant un comportement de vote socialement répréhensible (ici le fait de voter pour un "Grec ») entre les enjeux des élections locales et ceux des élections nationales a été observé à propos du vote blanc en France par (Zulfikarpasic 2001), dont les interlocuteurs avouent ne pas vouloir voter blanc lors de l'élection présidentielle pour laquelle les enjeux sont jugés trop importants.

98. Entretien avec la femme de Galip Galip, Komotini, août 2002.

99. Source $:$ http://www.parliament.gr/english/synthesh/mp.asp?MPID=426

100. Source $:$ http://www.parliament.gr/english/synthesh/mp.asp?MPID $=672$

101. Pour être exact, il faudrait y ajouter le village de Gökçeler (nom grec inconnu). « İskeçe ili 2004 seçim sonuçları » [Résultat des élections de 2004 dans le département de Xanthi], Gündem, 19/03/04. Par une bizarrerie administrative dont nous ignorons la raison, à côté des six municipalités que compte le département de Xanthi (Xanthi, Avdhira, Vistonidha, Mikis, Stavroupoli, Topiros), on dénombre les quatre villages mentionnés, qui ne sont rattachés à aucune municipalité.

102. Les municipalités ont été créées d'après la réforme Capodistrias (voir encadré) et sont en fait des regroupements de villages préexistants. Celui où est située la mairie donne son nom à la municipalité. Les trois derniers villages sont dans la même situation que ceux de l'échantillon de Xanthi.

103. De nombreux citoyens grecs d'Allemagne et de Chypre se sont également rendus aux urnes, mais nous ne savons pas dans quelle mesure cela a concerné les départements du Rhodope et de Xanthi (hormis pour les « musulmans » de Thrace résidant en Allemagne).

104. "Seçimde en kritik rolü Batı Trakya oynayacak » [La Thrace occidentale jouera un rôle critique dans les élections], op.cit.

105. C'est d'ailleurs celle qui est reprise dans le journal Gündem : d'après Rıza Kırlıdökme, il y aurait 26000 électeurs « turcs » dans le département de Xanthi, et 35000 dans celui du Rhodope. «Yine boynumuz bükük! » [Nous avons de nouveau courbé l'échine !], Gündem, 12/03/04. 


\section{RÉSUMÉS}

Western Thrace is a Greek region bordering Bulgaria and Turkey. Two of its administrative divisions (nomos) - Rhodopi and Xanthi - are mainly inhabited by members of the so-called 'Muslim minority'. Muslims remained in Greece after the 1924 compulsory population exchange with Turkey. Most of them are Turkish speakers and their electoral momentum has implications on the resonance of their political leaders' demand concerning the recognition by the Greek state of their 'Turkishness'. Since the beginning of the year 2000, in a context of European integration, claims linked to the 'Turkish' identity of the minority have been considered legitimate in the local political field, and are even taken over by some local 'Christian' politicians during electoral campaigns. These claims are still officially considered, however, as an element of national divide and consistently denounced in the courts by Greek state authorities. Despite the fact that both 'Greek' and 'Turkish' candidates suggest that voters have to vote along ethnic lines, the proportion of Muslim voters, that may theoretically allow the election of three 'Turkish' MPs, only one of them was elected in 2004. And yet, the electoral results show a proportionate increase in the votes for the 'Turkish' candidates. We have constituted a representative sample of the Muslim electorate in each nomos and made hypotheses in order to try to understand such a situation. These hypotheses deal with, on the one hand, the running rules of the local political field and the role of the 'Turkish' discourse in these rules; on the other hand, the Greek voting system's specificities.

\section{INDEX}

Keywords : Political Representation, Western Thrace, identitarianism, parliament, elections, Muslims, minorities, Greece, Greek Thrace 